\title{
Safe polycationic dendrimers as potent oral in vivo inhibitors of Mycobacterium tuberculosis: A new therapy to take down tuberculosis
}

Serge Mignani*a,b, Vishwa Deepak Tripathi, ${ }^{c, d}$, Dheerj Soam ${ }^{e}$, Rama Pati Tripathi*f, Swetarka Dase, Shriya Singhe, Ramakrishna Gandikota $^{c, d}$, Regis Laurent, ${ }^{c,}$, Andrii Karpus ${ }^{c, d}$, Anne-Marie Caminade ${ }^{c, d}$, Anke Steinmetz ${ }^{g}$, Arunava Dasgupta*h, Kishore Kumar Srivastava*h, Jean-Pierre Majoral ${ }^{*}, d$,

\section{Supplementary Information}

\section{Preparation of compounds of generation $0: 1 \mathrm{G0}, 2 \mathrm{G0}, 3 \mathrm{G0}$ and 1G0$, \mathrm{HCl}_{2}, 2 \mathrm{G0}, \mathrm{HCl}, 3 \mathrm{GG}, \mathrm{HCl}$}

Grafting 4-hydroxybenzylalcohol (2) on hexachlorocyclotriphosphazene core (1) (Cesium carbonate as base, room temperature, overnight) giving the compound 3 in 83\% yield as described in reference [Cavero E., Zablocka M, Caminade A.M., Majoral J.P. Design of Bisphosphonate-terminated Dendrimers. Eur. J. Org. Chem. 2010, 2759-2767], followed by addition of thionyl chloride (tetrahydrofuran, THF), room temperature, overnight) affords the corresponding benzyl chloride (4) in 93\% yield as described in reference above, which was further reacted with cyclic aliphatic amines (5a-c) under basic reaction condition such as cesium carbonate (room temperature, overnight) leading to 6 abcd (1G0, 2G0, $3 \mathrm{G} 0$ and $5 \mathrm{G} 0$ in $68 \%, 72 \%, 81 \%$ and $81 \%$ yield, respectively) corresponding to the incorporation of amines on the surface of dendrimers. These surfaces were further protonated using diethyl ether hydrogen chloride solution $\left(0^{\circ} \mathrm{C}\right.$, then slowly reach room temperature, overnight) to afford $1 \mathrm{G} 0_{, \mathrm{HCl}}, 2_{\mathrm{G} 0} \mathrm{HCl}, 3 \mathrm{G} 0_{, \mathrm{HCl}}$ and $5 \mathrm{G} 0_{, \mathrm{HCl}}$ (Scheme S1) in quantitative yield. 

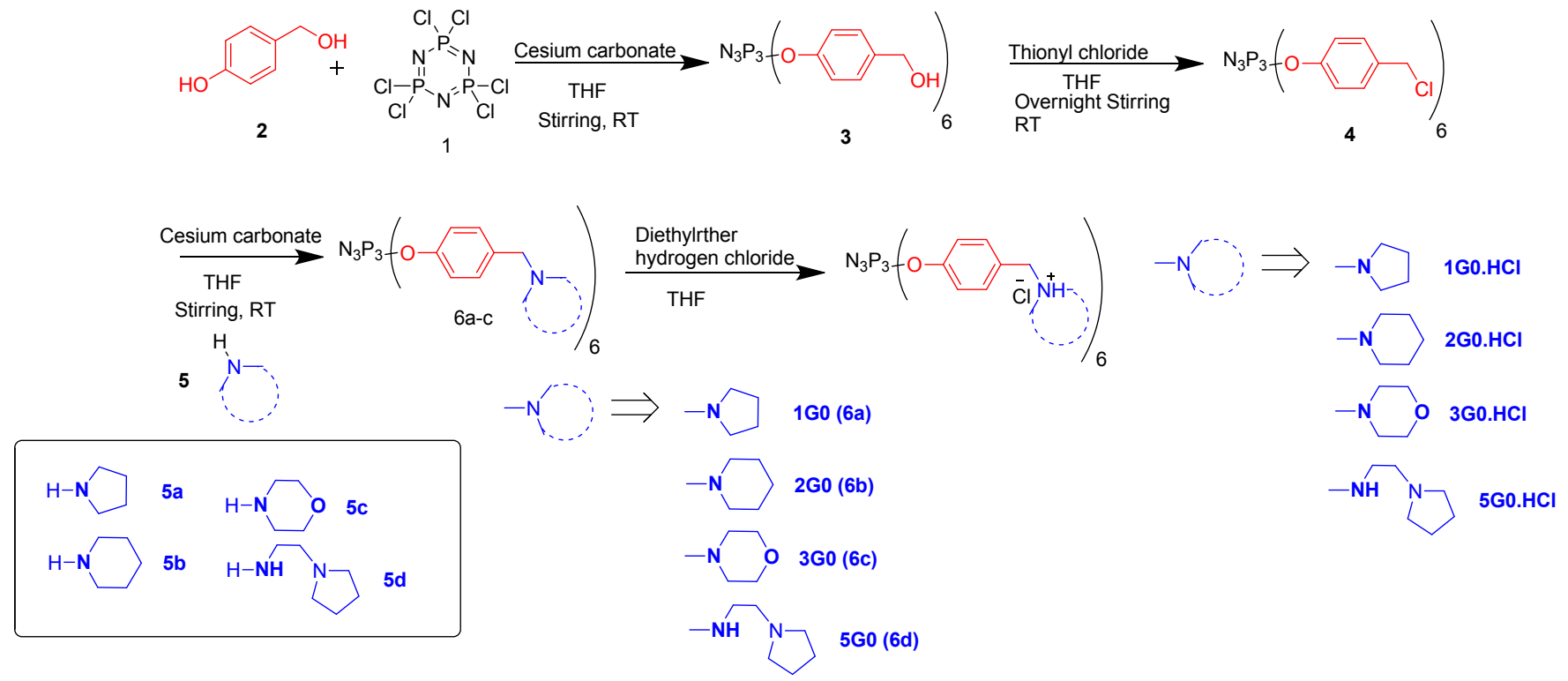

\section{Scheme S1}

\section{Compound 1G0.HCl}

Hexa\{4-(pyrrolidin-1-ylmethyl)phenoxy\}-1,3,5,2,4,6-triazatriphosphorine, hexa (6) hydrochloride

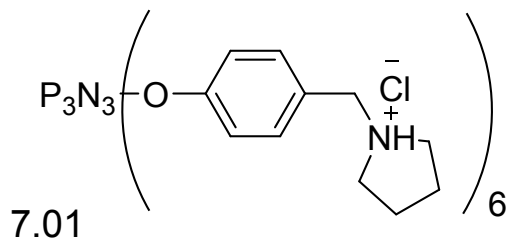

Molecular Formula: $\mathrm{C}_{66} \mathrm{H}_{90} \mathrm{Cl}_{6} \mathrm{~N}_{9} \mathrm{O}_{6} \mathrm{P}_{3} ; \mathrm{MW}: 1411$.

7.01

Molecular Formula: $\mathrm{C}_{66} \mathrm{H}_{90} \mathrm{Cl}_{6} \mathrm{~N}_{9} \mathrm{O}_{6} \mathrm{P}_{3}$; MW: $1411 .{ }^{1} \mathrm{H}$ NMR $\left(400 \mathrm{MHz}, \mathrm{D}_{2} \mathrm{O}\right) \delta=7.40(\mathrm{~d}, \mathrm{~J}=8 \mathrm{~Hz}, 12 \mathrm{H})$, $(\mathrm{d}, \mathrm{J}=8 \mathrm{~Hz}, 12 \mathrm{H}), 4.32(\mathrm{~s}, 12 \mathrm{H}), 3.36(\mathrm{~m}, 12 \mathrm{H}), 3.08(\mathrm{~m}, 12 \mathrm{H}), 2.04(\mathrm{~m}, 12 \mathrm{H}), 1.86(\mathrm{~m}, 12 \mathrm{H}) .{ }^{31} \mathrm{P}\left\{{ }^{1} \mathrm{H}\right\}$

$\operatorname{NMR}\left(161 \mathrm{MHz}, \mathrm{D}_{2} \mathrm{O}\right) \delta=8.80 .{ }^{13} \mathrm{C}\left\{{ }^{1} \mathrm{H}\right\} \operatorname{NMR}\left(100 \mathrm{MHz}, \mathrm{D}_{2} \mathrm{O}\right) \delta=150.43(\mathrm{~d}, \mathrm{~J}=4 \mathrm{~Hz}), 132.14,128.51,121.49,56.89,53.54,22.34$ 


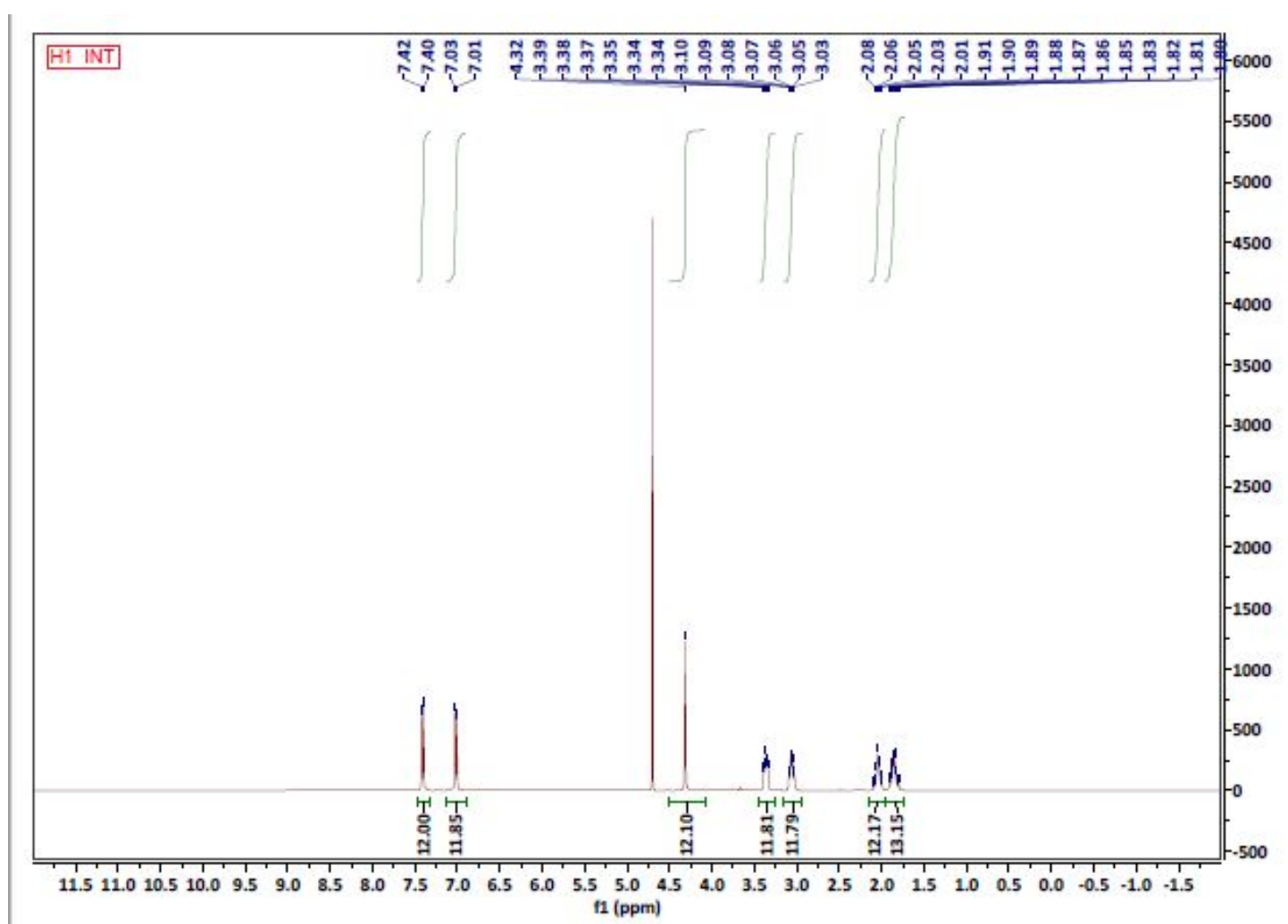




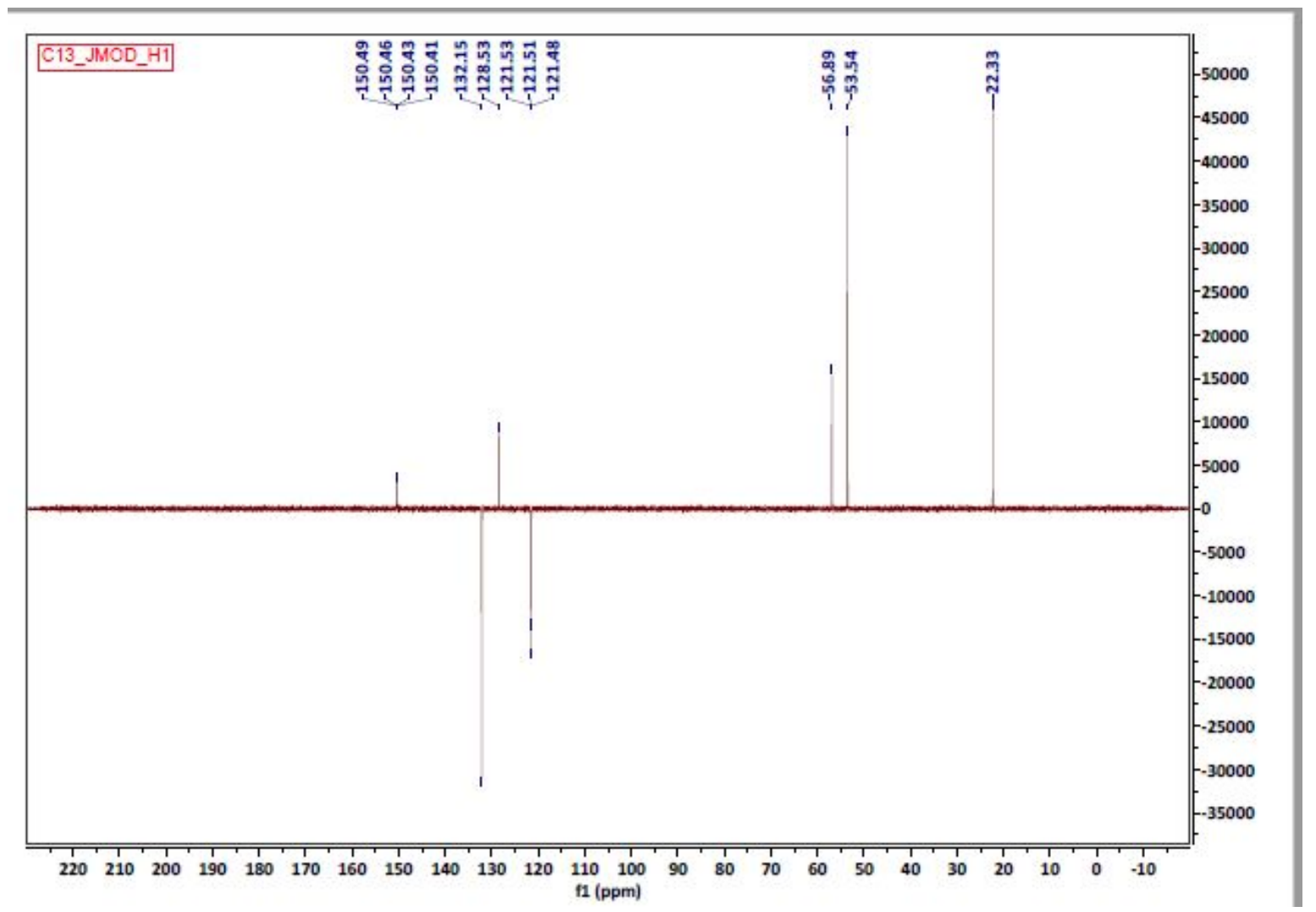




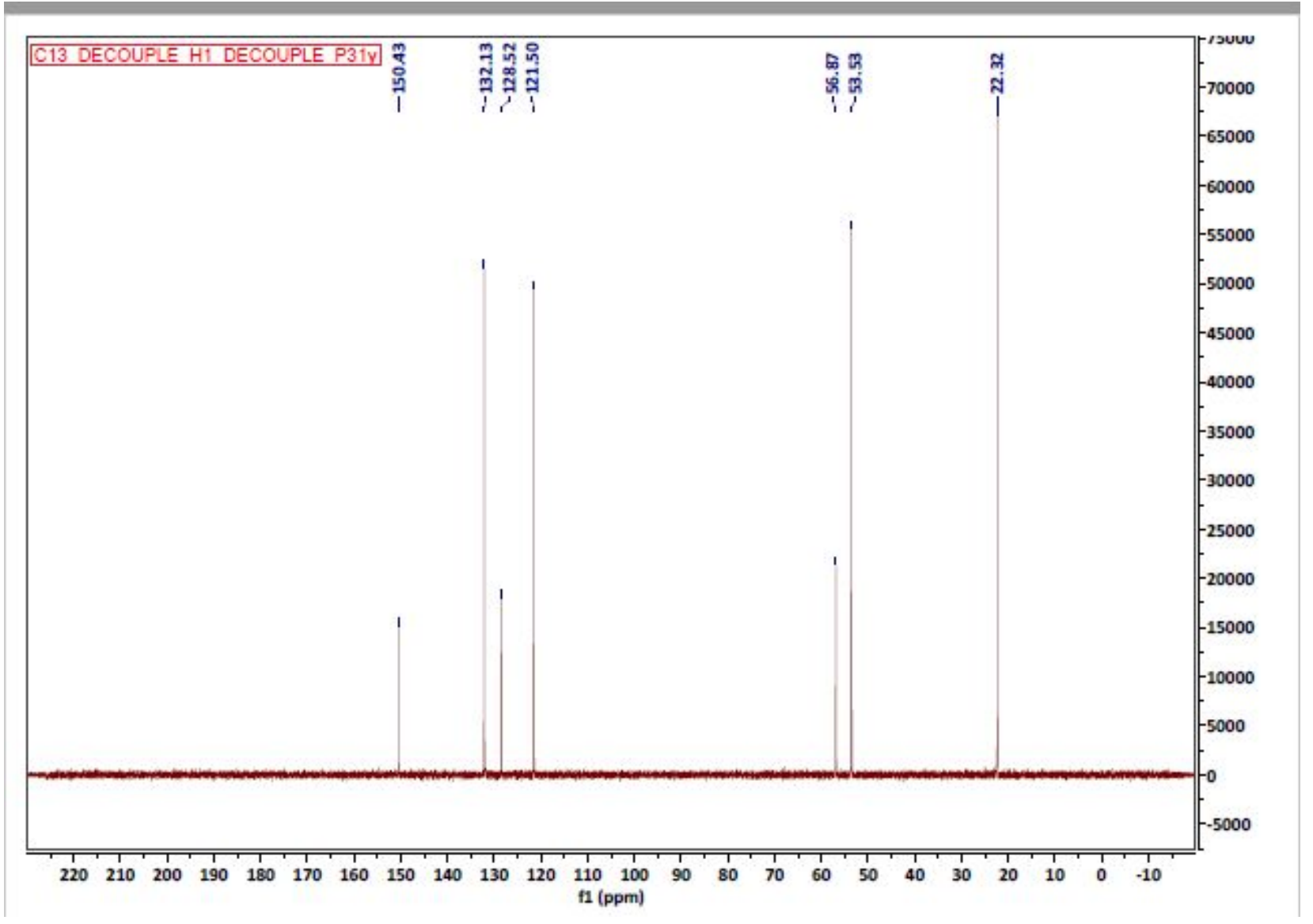




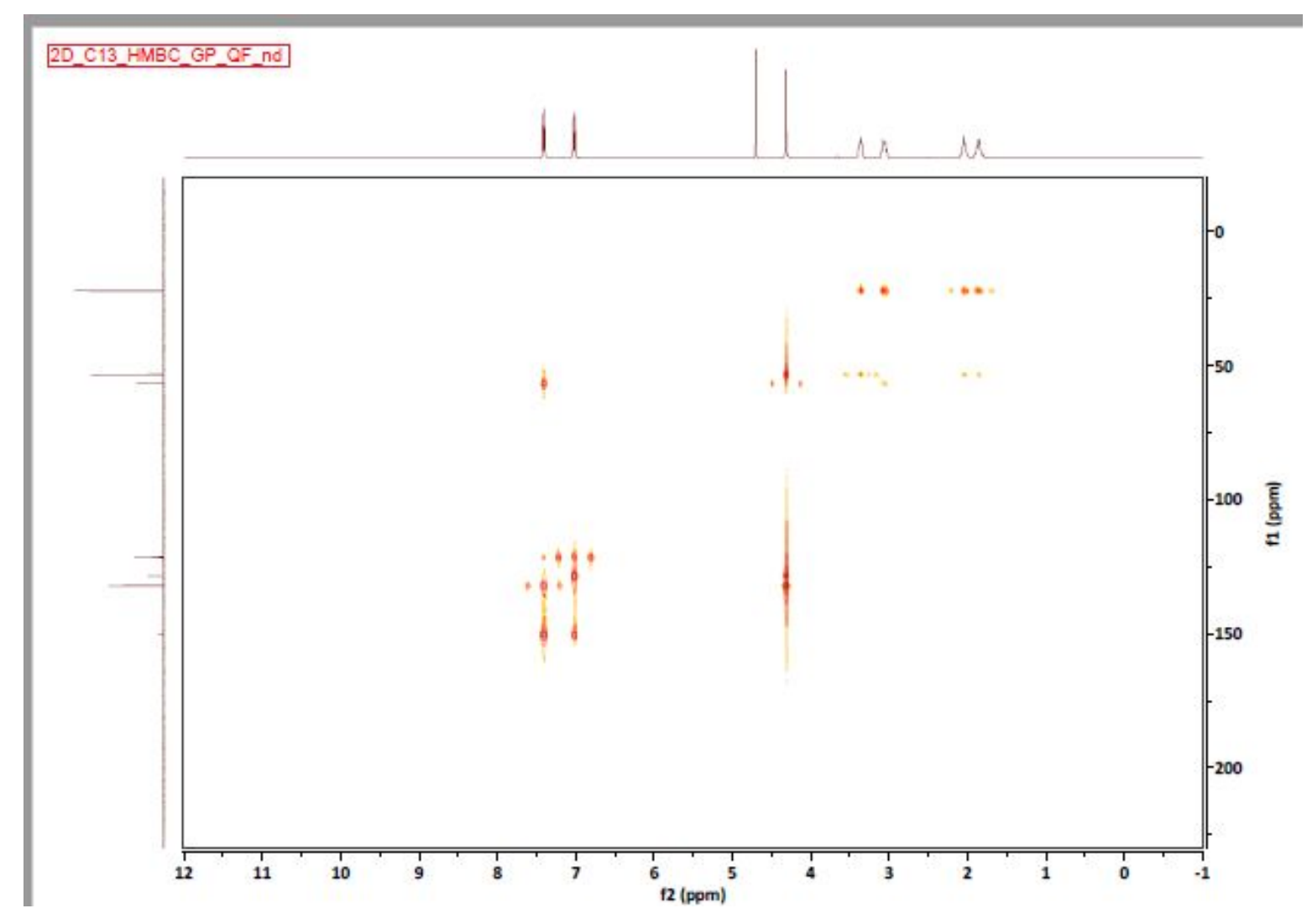




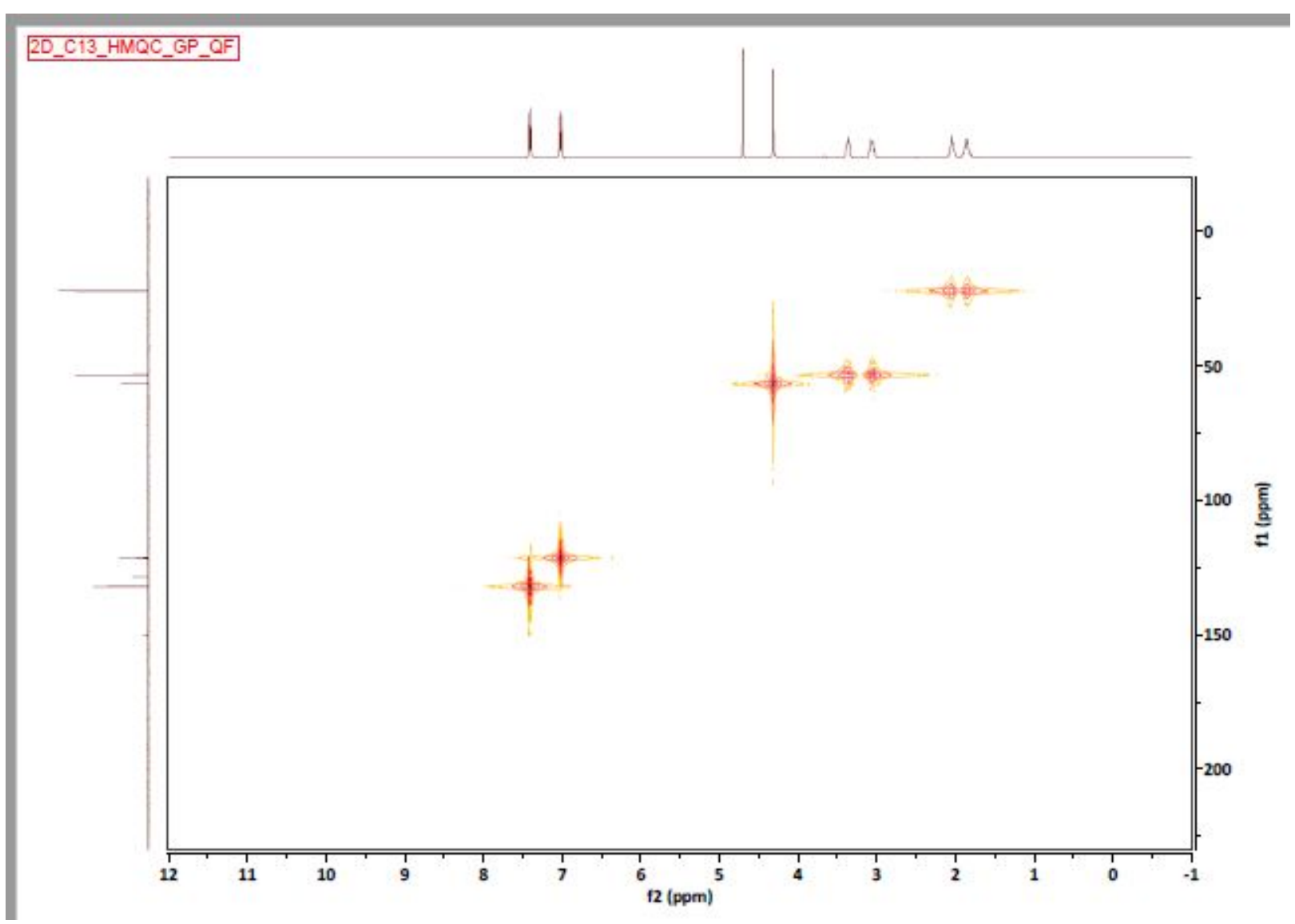

\section{Compound 1G0}

Hexa\{4-(pyrrolidin-1-ylmethyl)phenoxy\}-1,3,5,2,4,6-triazatriphosphorine

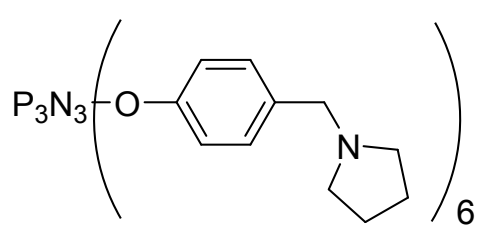

Molecular Formula: $\mathrm{C}_{66} \mathrm{H}_{84} \mathrm{~N}_{9} \mathrm{O}_{6} \mathrm{P}_{3}$; MW: 1192; ${ }^{1} \mathrm{H} \mathrm{NMR}(400 \mathrm{MHz}, \mathrm{CDCl} 3) \delta=7.15(\mathrm{~d}, \mathrm{~J}=8 \mathrm{~Hz}, 12 \mathrm{H}), 6.91(\mathrm{~d}, \mathrm{~J}=8 \mathrm{~Hz}, 12 \mathrm{H}), 3.57$ (s, 12H), 2.50-2.47 (m, 24H), 1.79-1.76 (m, 24H). (Yield = 68\%); ${ }^{31} \mathrm{P}\left\{{ }^{1} \mathrm{H}\right\} \mathrm{NMR}(161 \mathrm{MHz}, \mathrm{CDCl} 3) \delta=8.67(\mathrm{~s}) ;{ }^{13} \mathrm{C}\left\{{ }^{1} \mathrm{H}\right\}$ NMR $(100$ 
$\left.\mathrm{MHz}, \mathrm{CDCl}_{3}\right) \delta=149.55(\mathrm{~d}, \mathrm{~J}=4 \mathrm{~Hz}), 135.85,129.68,120.70,59.97,54.08,23.45 . \mathrm{MS}$ (ES+): Cal. Mass 1192.58; Obs. Mass $1192.59[\mathrm{M}]^{+}, 1193.59[\mathrm{M}+\mathrm{H}]^{+}$

\section{Compound 2G0, $\mathrm{HCl}$}

Hexa $\{4$-(piperidin-1-ylmethyl)phenoxy $\}$-1,3,5,2,4,6-triazatriphosphorine, hexa (6) hydrochloride

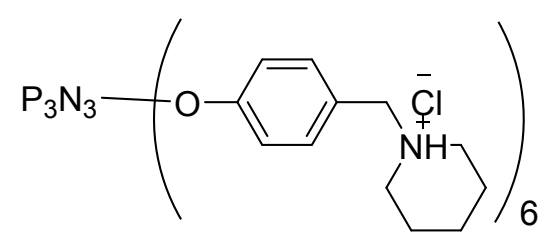

Molecular Formula: $\mathrm{C}_{72} \mathrm{H}_{102} \mathrm{Cl}_{6} \mathrm{~N}_{9} \mathrm{O}_{6} \mathrm{P}_{3}$; MW: 1495

Molecular Formula: $\mathrm{C}_{72} \mathrm{H}_{102} \mathrm{Cl}_{6} \mathrm{~N}_{9} \mathrm{O}_{6} \mathrm{P}_{3}$; MW: 1495. ${ }^{1} \mathrm{H}$ NMR $\left(400 \mathrm{MHz}, \mathrm{D}_{2} \mathrm{O}\right) \delta=7.40(\mathrm{~d}, \mathrm{~J}=8 \mathrm{~Hz}, 12 \mathrm{H}), 7.02(\mathrm{~d}, \mathrm{~J}=8 \mathrm{~Hz}, 12 \mathrm{H}), 4.22$ (s, 12H), $3.32(\mathrm{~m}, 12 \mathrm{H}), 2.83(\mathrm{~m}, 12 \mathrm{H}), 1.80(\mathrm{~m}, 12 \mathrm{H}), 1.75(\mathrm{~m}, 6 \mathrm{H}), 1.54(\mathrm{~m}, 12 \mathrm{H}), 1.31(\mathrm{~m}, 6 \mathrm{H}) .{ }^{31} \mathrm{P}\left\{{ }^{1} \mathrm{H}\right\} \mathrm{NMR}\left(161 \mathrm{MHz}, \mathrm{D}_{2} \mathrm{O}\right) \delta=$ 8.89. ${ }^{13} \mathrm{C}\left\{{ }^{1} \mathrm{H}\right\} \operatorname{NMR}\left(100 \mathrm{MHz}, \mathrm{D}_{2} \mathrm{O}\right) \delta=150.56(\mathrm{~d}, \mathrm{~J}=4 \mathrm{~Hz}), 133.07,126.85,121.43,59.38,52.69,22.63,21.00$ 


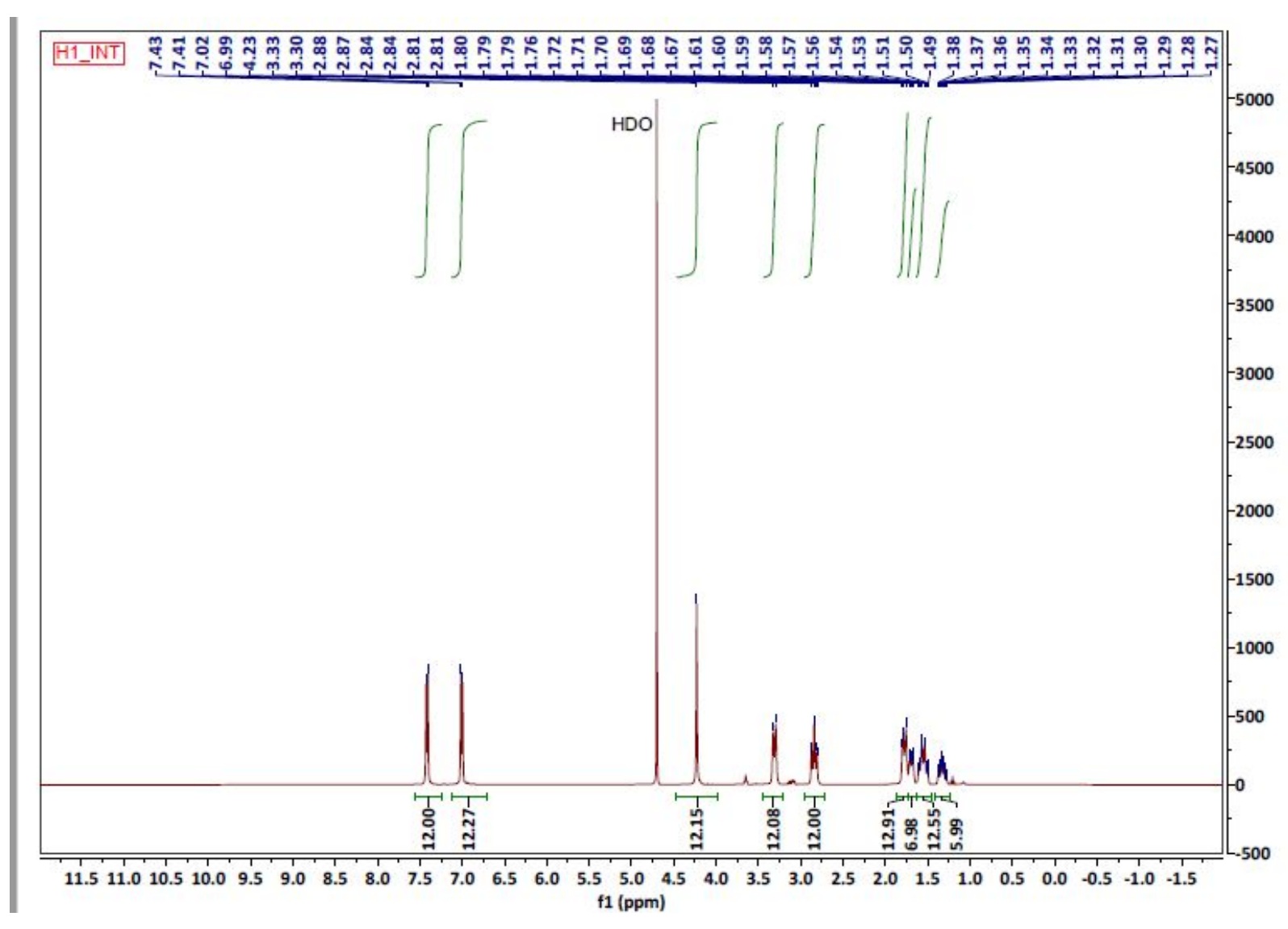




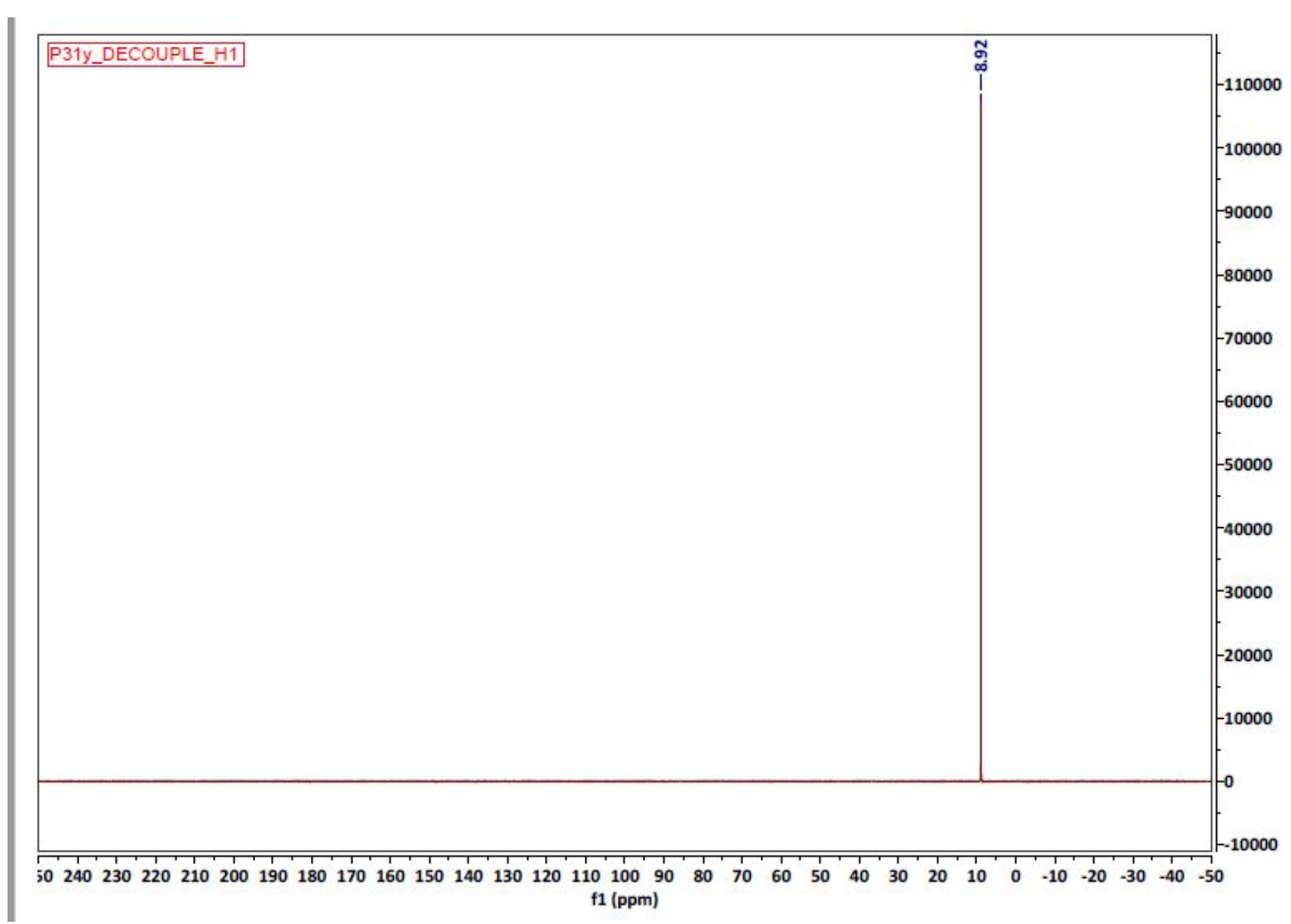




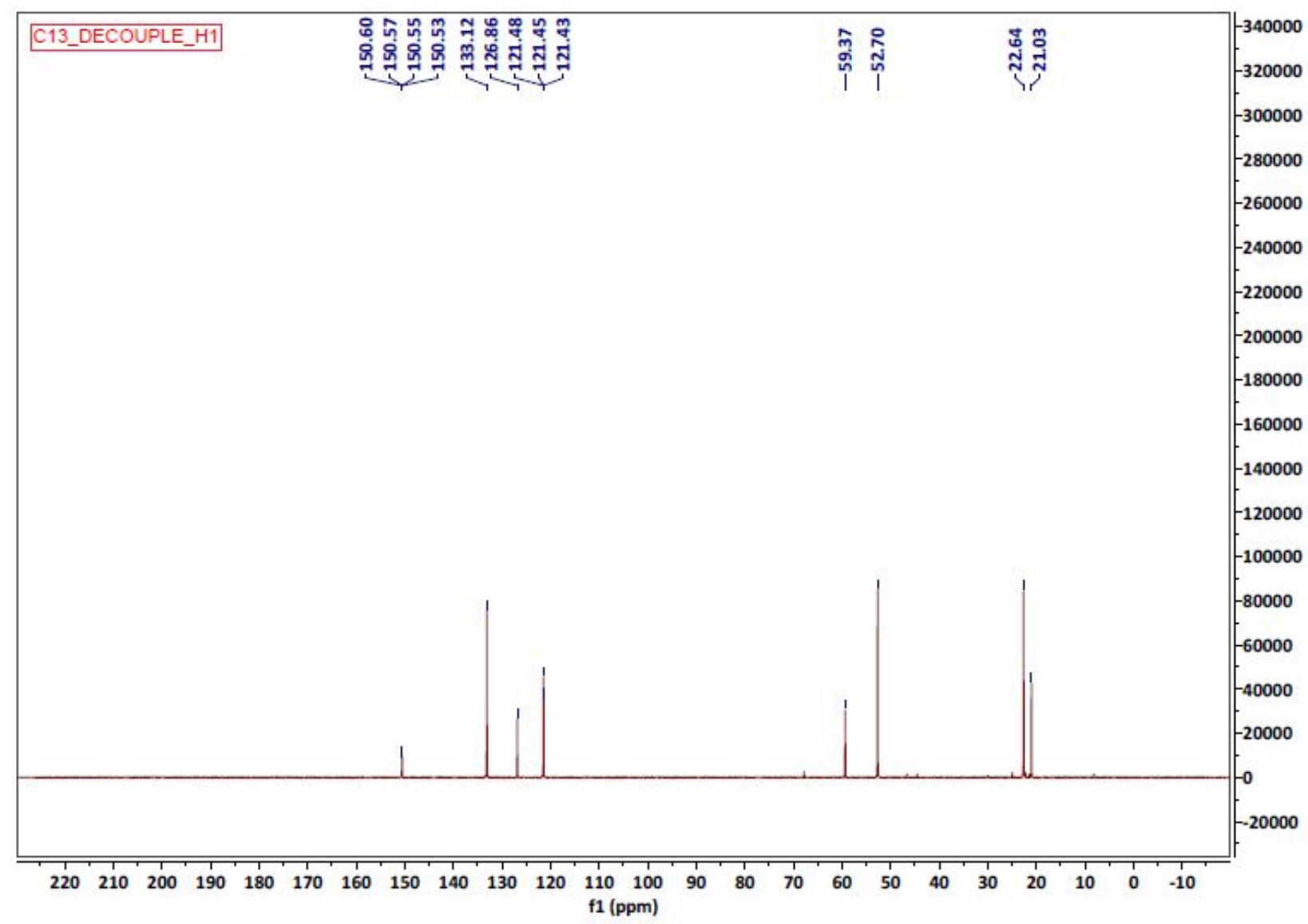




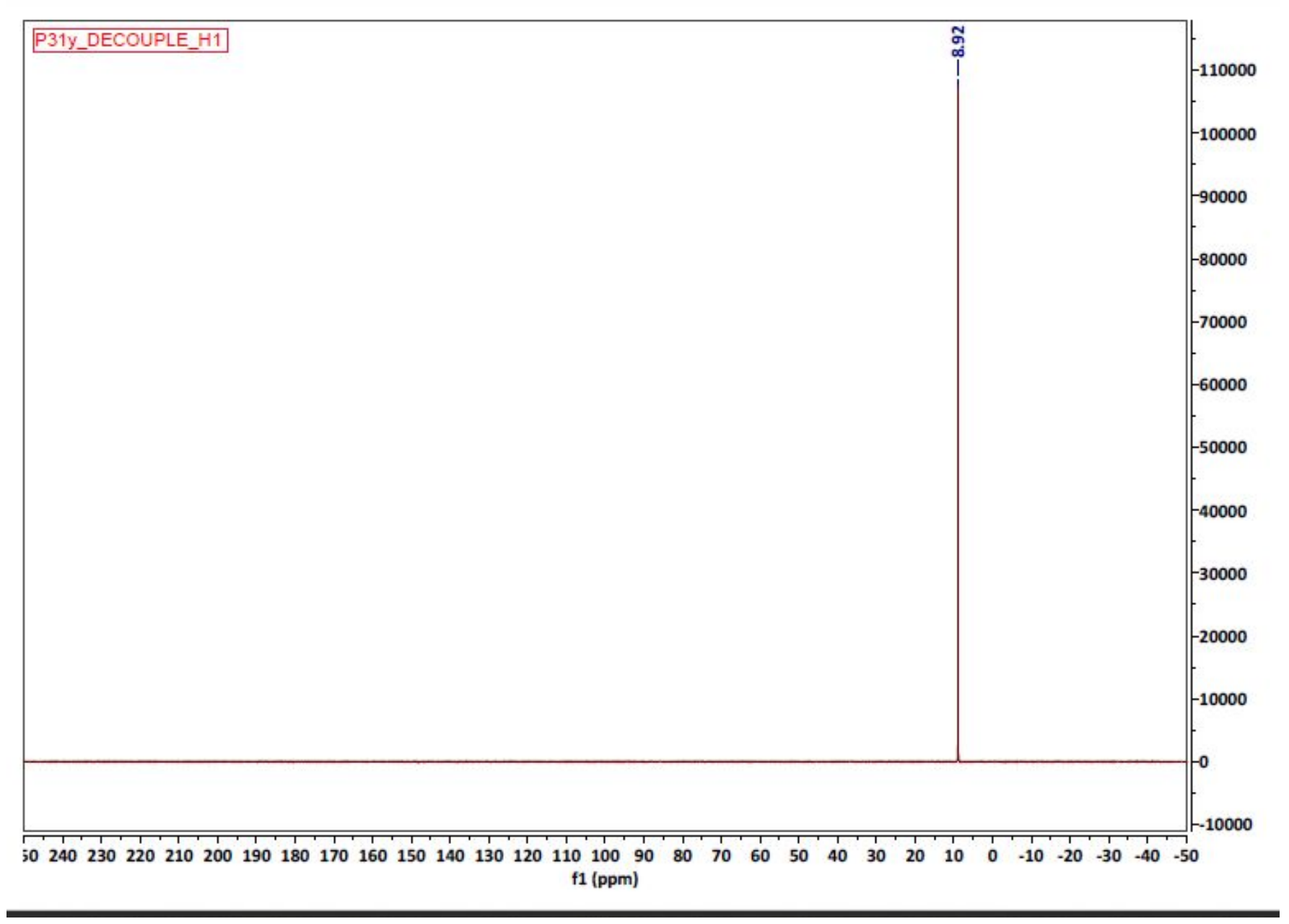




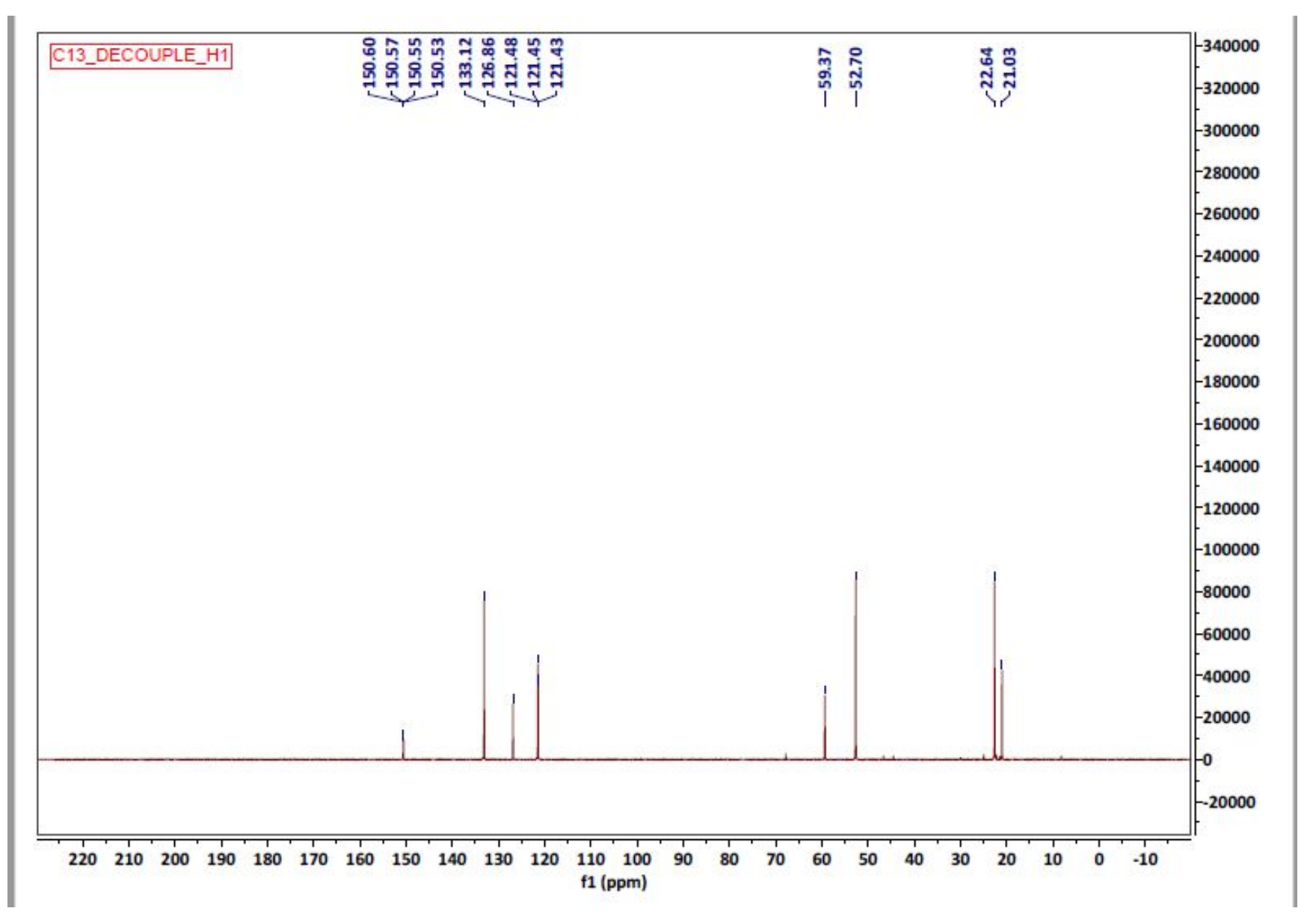




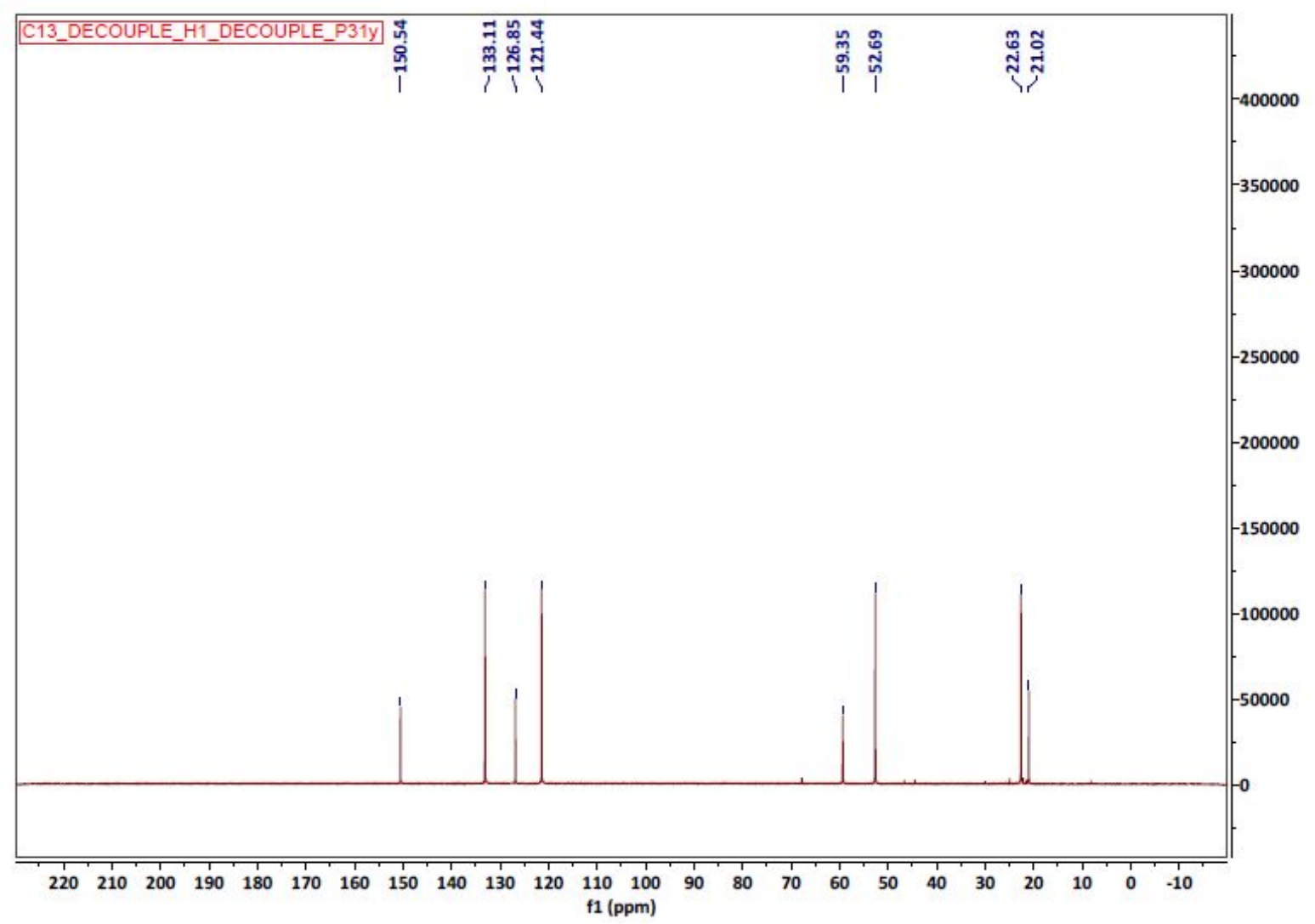




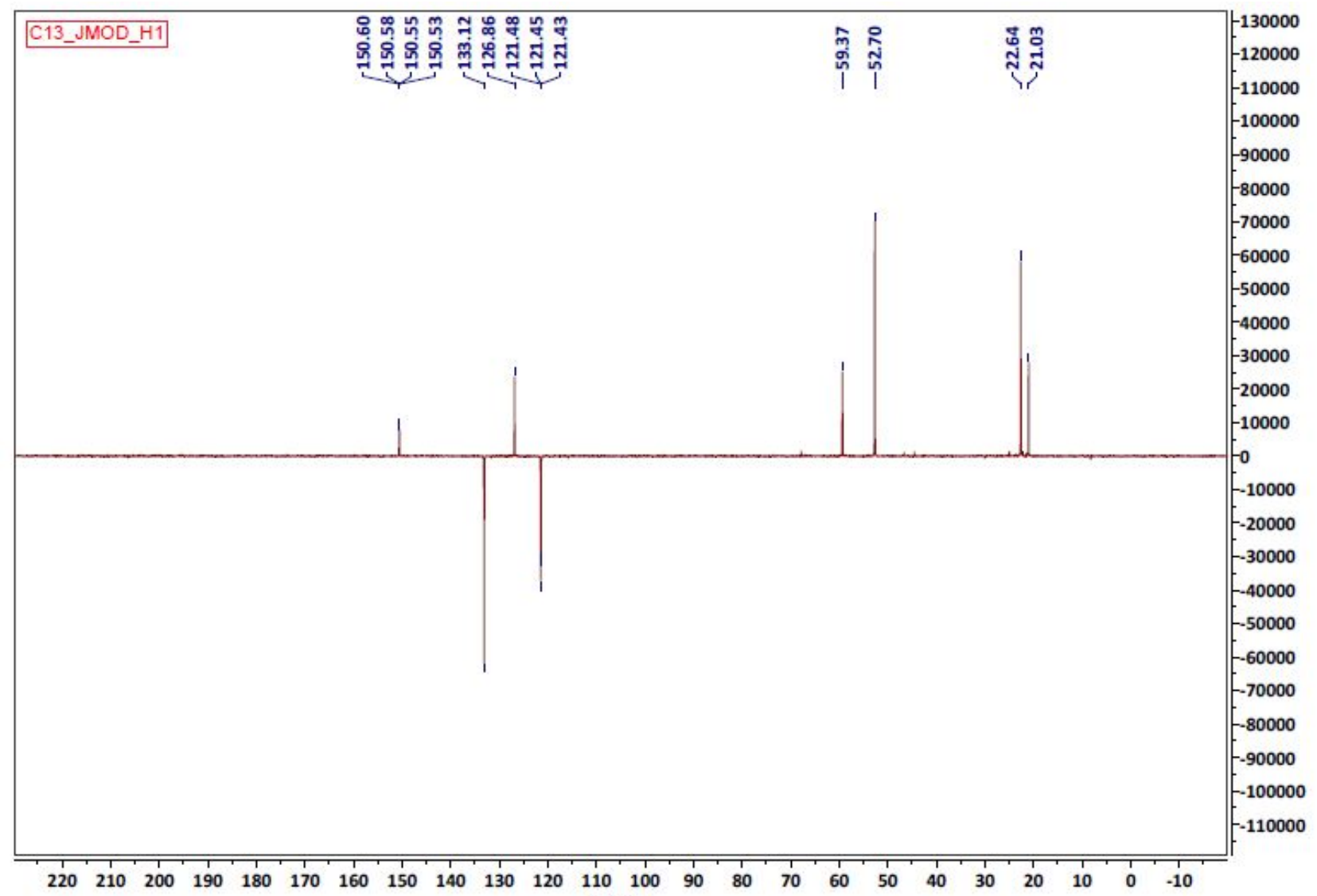

f1 (ppm) 


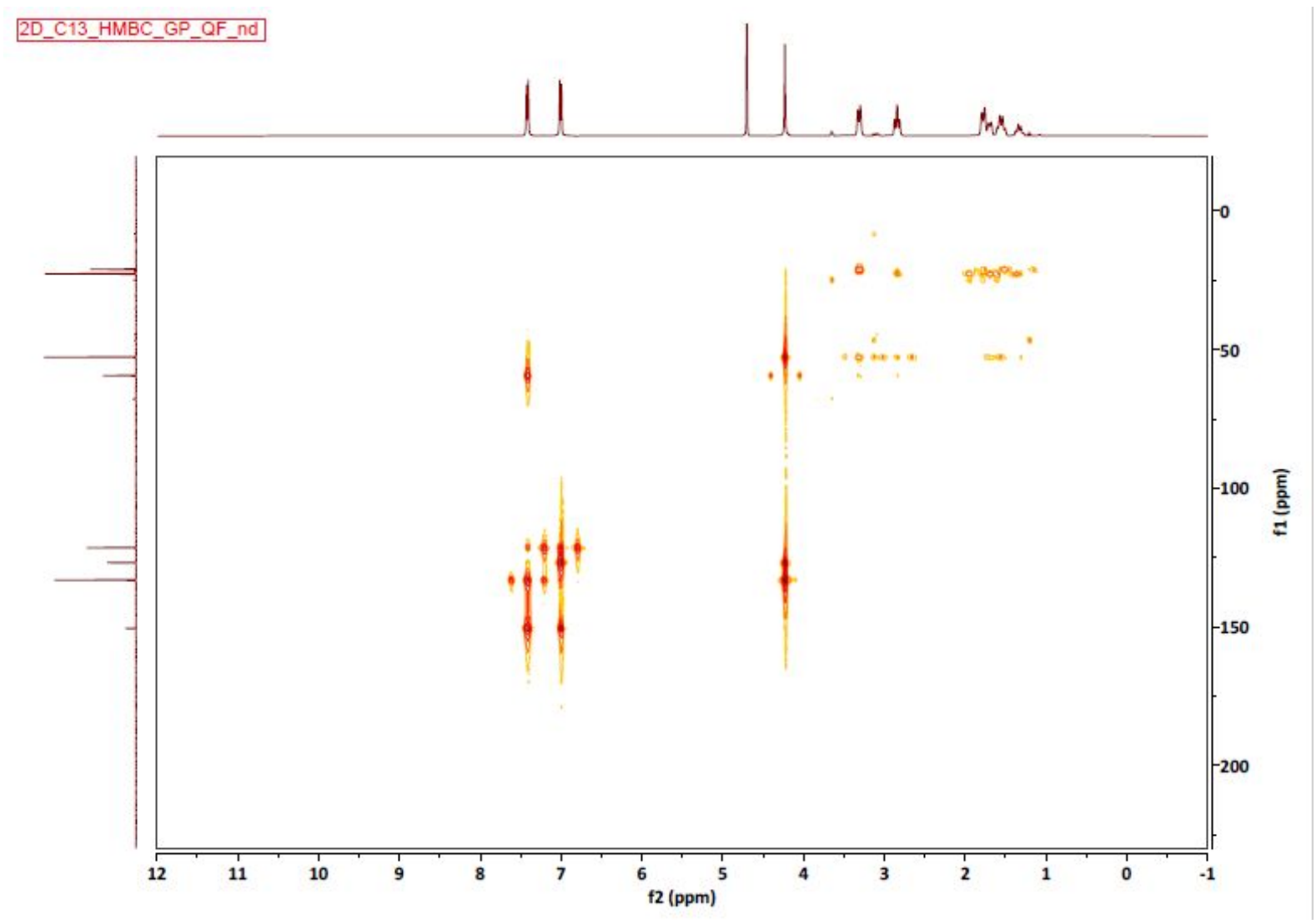




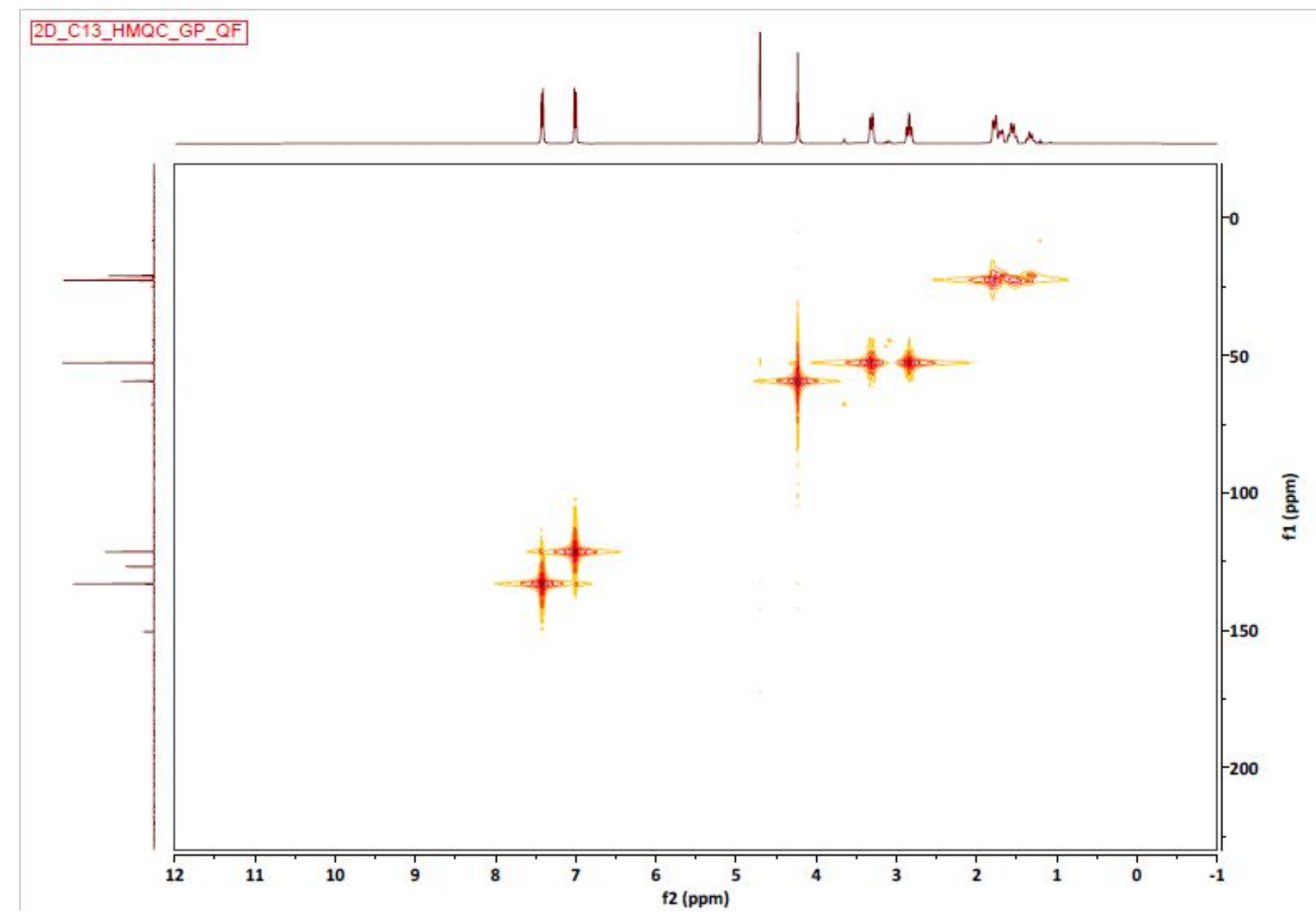

\section{Compound 2G0}

Hexa $\{4$-(piperidin-1-ylmethyl)phenoxy $\}-1,3,5,2,4,6$-triazatriphosphorine

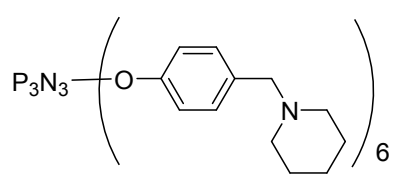

Molecular Formula: $\mathrm{C}_{72} \mathrm{H}_{96} \mathrm{~N}_{9} \mathrm{O}_{6} \mathrm{P}_{3}$; MW: 1276; ${ }^{1} \mathrm{H} \mathrm{NMR}(300 \mathrm{MHz}, \mathrm{CDCl} 3) \delta=7.13(\mathrm{~d}, \mathrm{~J}=8 \mathrm{~Hz}, 12 \mathrm{H}), 6.90(\mathrm{~d}, \mathrm{~J}=8 \mathrm{~Hz}, 12 \mathrm{H}), 3.43$ $(\mathrm{s}, 12 \mathrm{H}), 2.35(\mathrm{~s}, 24 \mathrm{H}), 1.60-1.53(\mathrm{~m}, 24 \mathrm{H}), 1.44-1.43(\mathrm{~m}, 12 \mathrm{H}) .($ Yield $=72 \%) ;{ }^{31} \mathrm{P}\left\{{ }^{1} \mathrm{H}\right\} \mathrm{NMR}(121 \mathrm{MHz}, \mathrm{CDCl} 3) \delta=8.69(\mathrm{~s}) ;{ }^{13} \mathrm{C}\left\{{ }^{1} \mathrm{H}\right\}$ 
$\operatorname{NMR}(75 \mathrm{MHz}, \mathrm{CDCl} 3) \delta=149.61$ (d, J = 4 Hz), 135.02, 130.00, 120.67, 63.14, 54.41, 25.94, 24.35. MS (ES+): Cal. Mass 1276.68; Obs. Mass $1276.68[\mathrm{M}]^{+}, 1277.69[\mathrm{M}+\mathrm{H}]^{+}$

\section{Compound 3G0, ${ }_{\text {HCl: }}$}

Hexa\{4-(morpholin-1-ylmethyl)phenoxy\}-1,3,5,2,4,6-triazatriphosphorine, hexa (6) hydrochloride

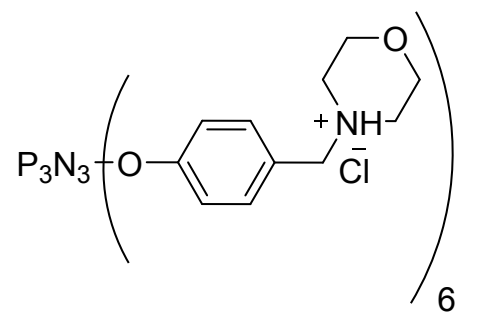

Molecular formula: $\mathrm{C}_{66} \mathrm{H}_{90} \mathrm{Cl}_{6} \mathrm{~N}_{9} \mathrm{O}_{12} \mathrm{P}_{3}$; $\mathrm{MW}: 1507$.

\section{Compound 3G0}

Hexa $\{4$-(morpholin-1-ylmethyl)phenoxy $\}$-1,3,5,2,4,6-triazatriphosphorine

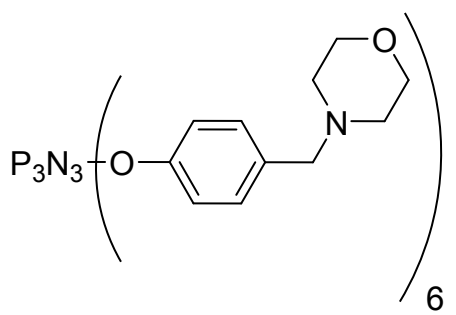

Molecular formula: $\mathrm{C}_{66} \mathrm{H}_{84} \mathrm{~N}_{9} \mathrm{O}_{12} \mathrm{P}_{3}$; MW: 1288. ${ }^{1} \mathrm{H}$ NMR (300 MHz, CDCl3) $\delta=7.16(\mathrm{~d}, \mathrm{~J}=8 \mathrm{~Hz}, 12 \mathrm{H}), 6.92(\mathrm{~d}, \mathrm{~J}=8 \mathrm{~Hz}, 12 \mathrm{H}), 3.88$ (brt, J = $6 \mathrm{~Hz}, 24 \mathrm{H}), 3.45(\mathrm{~s}, 12 \mathrm{H}), 2.41(\mathrm{t}, \mathrm{J}=6 \mathrm{~Hz}, 24 \mathrm{H}) .($ Yield $=81 \%) .{ }^{31} \mathrm{P}\left\{{ }^{1} \mathrm{H}\right\} \mathrm{NMR}(121 \mathrm{MHz}, \mathrm{CDCl} 3) \delta=8.60(\mathrm{~s}) ;{ }^{13} \mathrm{C}\left\{{ }^{1} \mathrm{H}\right\}$ NMR $(75 \mathrm{MHz}, \mathrm{CDCl} 3) \delta=149.68$ (d, J = 4 Hz), 134.47, 130.01, 120.73, 66.96, 62.76, 53.59.

\section{Preparation of compounds of generation 0: 5G0 and 5G0,}




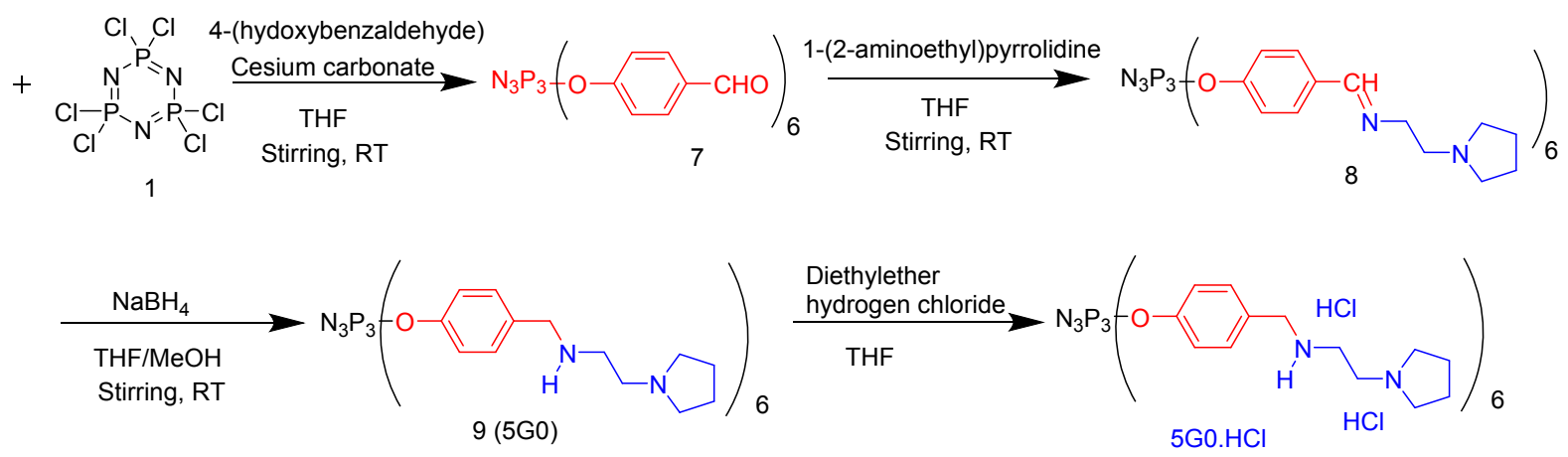

\section{Scheme S2}

Dendrimer 7 was treated with 1-(2-aminoethyl)pyrrolidine (THF, room temperature, one night) to give 8 . Then, 8 was treated with $\mathrm{NaBH}_{4}$ (THF-methanol mixture, room temperature, one night) to give 9 (5G0) which was protonated (diethylether, hydrogen chloride) to afford $5 \mathrm{G} 0 . \mathrm{HCl}$

The compound 7 was prepared according to the reference [Padie, C.; Maszewska, M.; Majchrzak, K.; Nawrot, B.; Caminade, A.M.; Majoral, J.P. Polycationic phosphorus dendrimers: synthesis, characterization, study of cytotoxicity, complexation of DNA, and transfection experiments, New J. Chem., 2009, 33, 318-326. DOI: 10.1039/B815259D]

\section{Compound 5G0 ${ }_{\mathrm{HCl}}$}

Hexa\{4-(((2-(pyrrolidin-1-yl)ethyl)amino)methyl)phenoxy\}-1,3,5,2,4,6-triazatriphosphorine, dodeca (12) hydrochloride

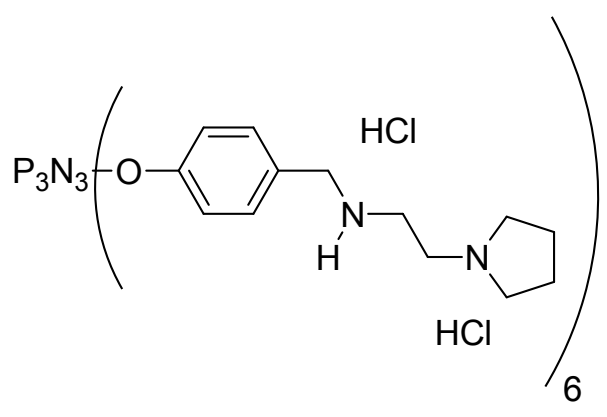

Molecular formula: $\mathrm{C}_{78} \mathrm{H}_{126} \mathrm{Cl}_{12} \mathrm{~N}_{9} \mathrm{O}_{12} \mathrm{P}_{3}$, MW: 1900 .

Compound 5G0: Hexa\{4-(((2-(pyrrolidin-1-yl)ethyl)amino)methyl)phenoxy\}-1,3,5,2,4, 6-triazatriphosphorine 


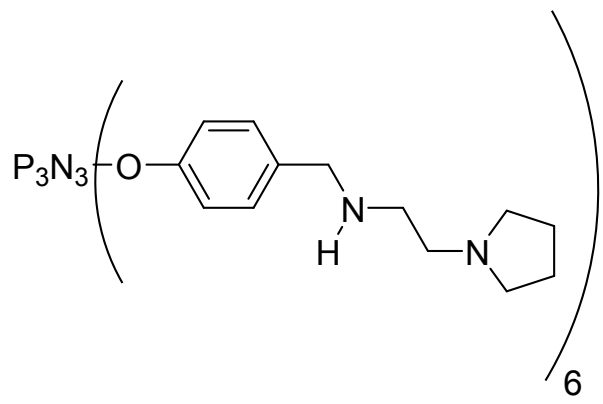

Molecular formula: $\mathrm{C}_{78} \mathrm{H}_{114} \mathrm{~N}_{9} \mathrm{O}_{12} \mathrm{P}_{3}$; MW: 1463. ${ }^{1} \mathrm{H}$ NMR (300 MHz, CDCl3) $\delta=7.15(\mathrm{~d}, \mathrm{~J}=8 \mathrm{~Hz}, 12 \mathrm{H}), 6.92(\mathrm{~d}, \mathrm{~J}=8 \mathrm{~Hz}, 12 \mathrm{H}), 3.76$ (s, 12H), $2.72(\mathrm{t}, \mathrm{J}=6 \mathrm{~Hz}, 12 \mathrm{H}), 2.61(\mathrm{t}, \mathrm{J}=6 \mathrm{~Hz}, 12 \mathrm{H}), 2.50$ (br t, 24H), 2.10 (br s, 6H), 1.75 (br t, 24H). (Yield = 81\%). ${ }^{11} \mathrm{P}\left\{{ }^{1} \mathrm{H}\right\} \mathrm{NMR}$ $(121 \mathrm{MHz}, \mathrm{CDCl} 3) \delta=8.58(\mathrm{~s}) .{ }^{13} \mathrm{C}\left\{{ }^{1} \mathrm{H}\right\} \operatorname{NMR}\left(100 \mathrm{MHz}, \mathrm{CDCl}_{3}\right) \delta=149.60(\mathrm{~d}, \mathrm{~J}=4 \mathrm{~Hz}), 136.97,129.00,120.80,55.91,54.21$,

$53.44,47.88,23.45$.

\section{Preparation of compounds of generation 1 (6G1, 7G1, 8G1, 9G1, 10G1, 11G1 and 12G1) and (6G,}

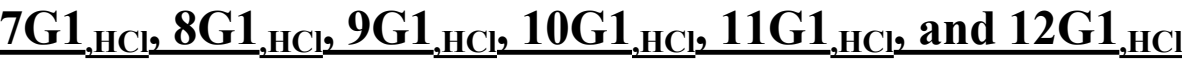

The dendrimer 6 was grafted with the diamines 7a-g under basic reaction conditions (DIPEA, room temperature,overnight) to afford $6 \mathrm{G} 1-12 \mathrm{G} 1$ in $71-79 \%$ yield. These neutral dendrimers were further converted to their respective cationic dendrimers $6 \mathrm{G} 1_{, \mathrm{HCl}}{ }^{12 \mathrm{G} 1_{, \mathrm{HCl}}}$ by protonation with hydrogen chloride in diethylether, in $\mathrm{THF}$ at $0^{\circ} \mathrm{C}$, reaching slowly room temperature overnight (quantitative yield) (Scheme S3). 

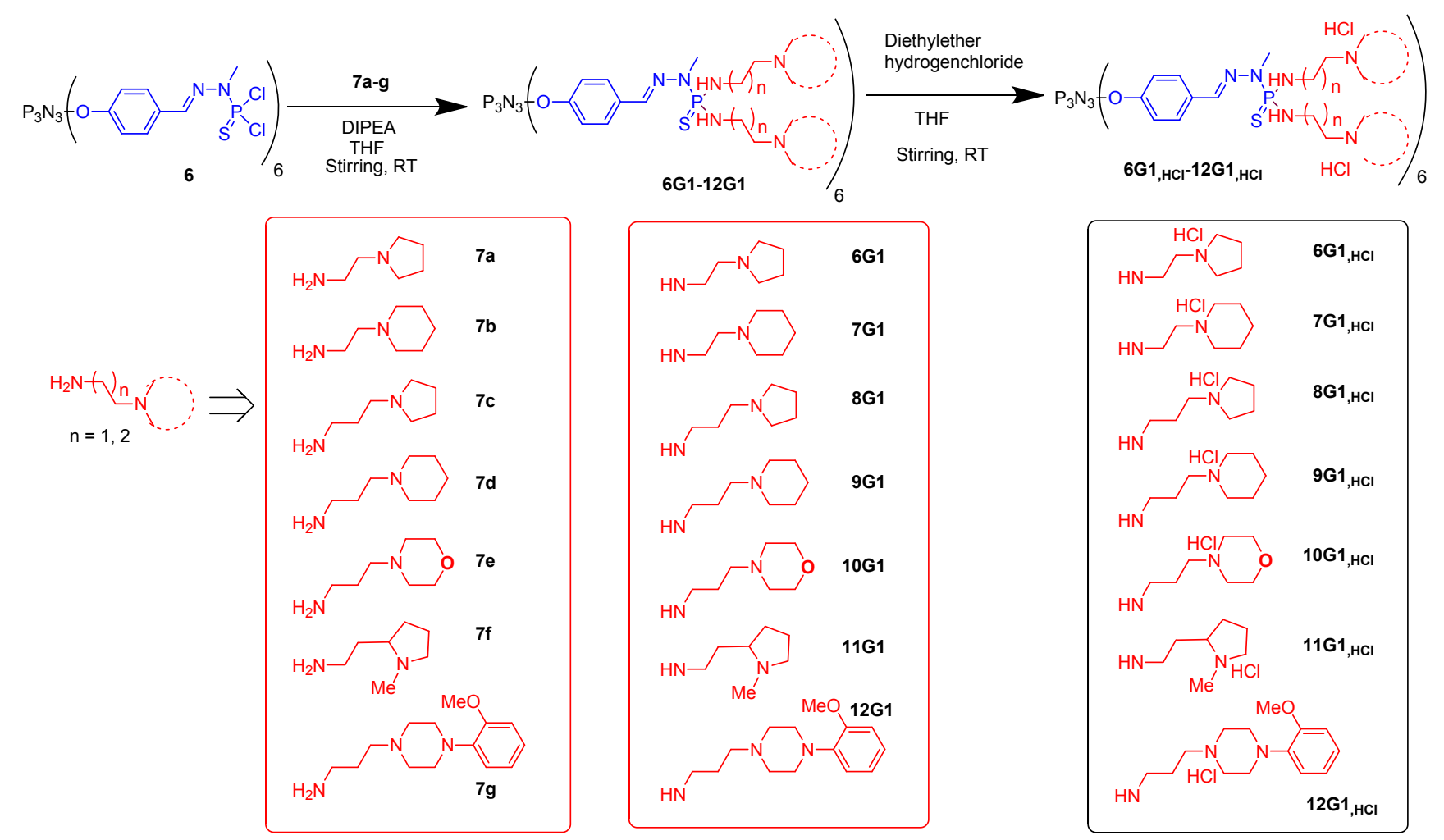

\section{Scheme S3}

The compound 6 has been prepared according to the procedure described in the Publications [a) Slany M., Bardají M., Casanove M.J., Caminade A.M., Majoral J.P., Chaudret B. Dendrimer surface chemistry. An easy access to polyphosphines and their gold complexes. J. Am. Chem. Soc. 1995, 117, 9764; b) Launay N., Caminade A.M., Majoral J.P. Synthesis of bowl-shaped dendrimers from generation 1 to generation 8. J. Organomet. Chem. 1997, 529, 51]

\section{Compound 6G1, $\mathrm{HCl}$}

$\{[\{N, N-(2-($ pyrrolidin-1-yl)ethanamine $\}$-phosphonothioate $]-\{4-((2-m e t h y l h y d r a z o n o) m e t h y l)\}$ phenoxy $\}-1,3,5,2,4,6-$

triazatriphosphorine, dodeca (12) hydrochloride 


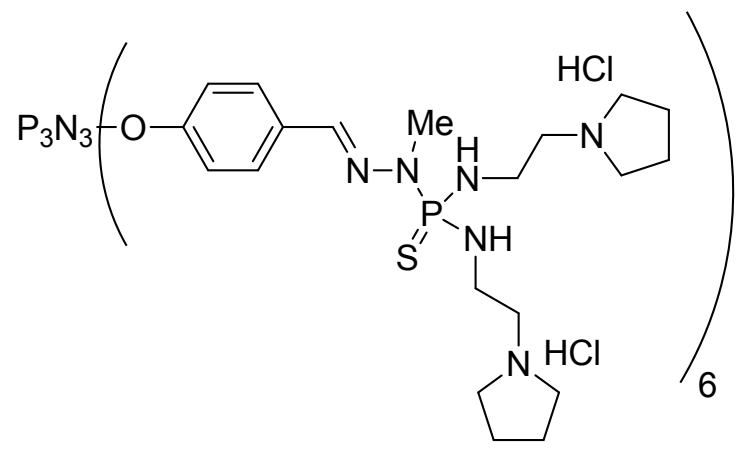

Molecular Formula: $\mathrm{C}_{120} \mathrm{H}_{216} \mathrm{Cl}_{12} \mathrm{~N}_{39} \mathrm{O}_{6} \mathrm{P}_{9} \mathrm{~S}_{6}$; MW: 3198. ${ }^{1} \mathrm{H}$ NMR $\left(400 \mathrm{MHz}, \mathrm{D}_{2} \mathrm{O}\right) \delta=7.79(\mathrm{~s}, 6 \mathrm{H}), 7.57(\mathrm{~d}, \mathrm{~J}=8 \mathrm{~Hz}, 12 \mathrm{H}), 6.91(\mathrm{~d}, \mathrm{~J}=$ $8 \mathrm{~Hz}, 12 \mathrm{H}), 3.7-3.5(\mathrm{~m}, 24 \mathrm{H}), 3.35-3.20(\mathrm{~m}, 48 \mathrm{H}), 3.16(\mathrm{~d}, \mathrm{~J}=10 \mathrm{~Hz}, 18 \mathrm{H}), 3.05-2.85(\mathrm{~m}, 24 \mathrm{H}), 1.96(\mathrm{~m}, 24 \mathrm{H}), 1.81(\mathrm{~m}, 24 \mathrm{H}) .{ }^{31} \mathrm{P}\left\{{ }^{1} \mathrm{H}\right\}$ $\operatorname{NMR}\left(161 \mathrm{MHz}, \mathrm{D}_{2} \mathrm{O}\right) \delta=70.21,9.13 .{ }^{13} \mathrm{C}\left\{{ }^{1} \mathrm{H}\right\} \mathrm{NMR}\left(100 \mathrm{MHz}, \mathrm{D}_{2} \mathrm{O}\right) \delta=149.94(\mathrm{~d}, \mathrm{~J}=4 \mathrm{~Hz}), 139.42(\mathrm{~d}, \mathrm{~J}=14 \mathrm{~Hz}), 133.08,128.24$, 121.32, 55.24 (d, J = $7 \mathrm{~Hz}), 54.46,37.35,31.99(\mathrm{~d}, \mathrm{~J}=10 \mathrm{~Hz}), 22.53$.

\section{Compound 6G1}

$\{[\{N, N$-(2-(pyrrolidin-1-yl)ethanamine $\}$-phosphonothioate $]-\{4-((2-m e t h y l h y d r a z o n o) m e t h y l)\}$ phenoxy $\}-1,3,5,2,4,6-$

triazatriphosphorine

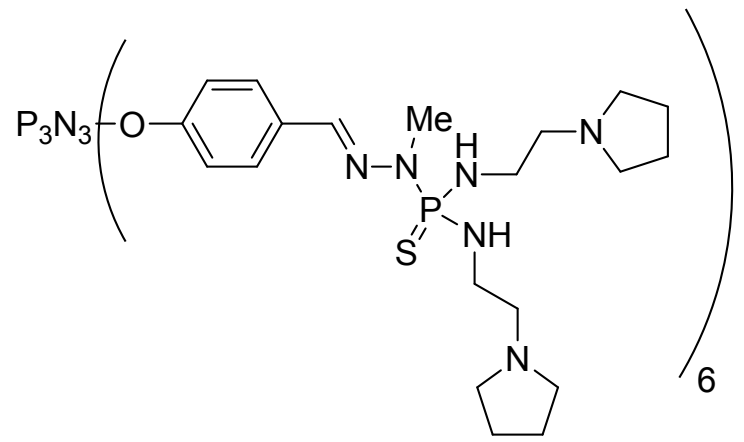

Molecular formula: $\mathrm{C}_{120} \mathrm{H}_{204} \mathrm{~N}_{39} \mathrm{O}_{6} \mathrm{P}_{9} \mathrm{~S}_{6}$; MW: 2760. ${ }^{1} \mathrm{H}$ NMR $(300 \mathrm{MHz}, \mathrm{CDCl} 3) \delta=7.49(\mathrm{~s}, 6 \mathrm{H}), 7.46(\mathrm{~d}, \mathrm{~J}=9.0 \mathrm{~Hz}, 12 \mathrm{H}), 6.93(\mathrm{~d}, \mathrm{~J}=$ $9.0 \mathrm{~Hz}, 12 \mathrm{H}), 4.11-4.04(\mathrm{~m}, 12 \mathrm{H}), 3.16(\mathrm{~d}, \mathrm{~J}=12 \mathrm{~Hz}, 18 \mathrm{H}), 3.12-2.94(\mathrm{~m}, 24 \mathrm{H}), 2.67-2.54(\mathrm{~m}, 24 \mathrm{H}), 2.49(\mathrm{brs}, 48 \mathrm{H}), 1.72(\mathrm{brs}, 48 \mathrm{H})$. (Yield $=71 \%) ;{ }^{31} \mathrm{P}\left\{{ }^{1} \mathrm{H}\right\}$ NMR $(121 \mathrm{MHz}, \mathrm{CDCl} 3) \delta=68.55,8.24 ;{ }^{13} \mathrm{C}\left\{{ }^{1} \mathrm{H}\right\} \mathrm{NMR}(75 \mathrm{MHz}, \mathrm{CDCl} 3) \delta=150.56,135.77(\mathrm{~d}, \mathrm{~J}=20.6 \mathrm{~Hz})$, 
132.89, 127.44, 121.03, 56.17 (d, J = 13.3 Hz), 53.77, 39.79, 30.83 (d, J = 14 Hz), 23.54. MS (ES+): Cal. Mass 2760.29; Obs. Mass $2760.33[\mathrm{M}]^{+}$

\section{Compound 7G1,HCl}

$\{[\{N, N$-(2-(piperidin-1-yl)ethanamine $\}$-phosphonothioate $]-\{4-((2-m e t h y l h y d r a z o n o) m e t h y l)\}$ phenoxy $\}-1,3,5,2,4,6-$ triazatriphosphorine, dodeca (12) hydrochloride

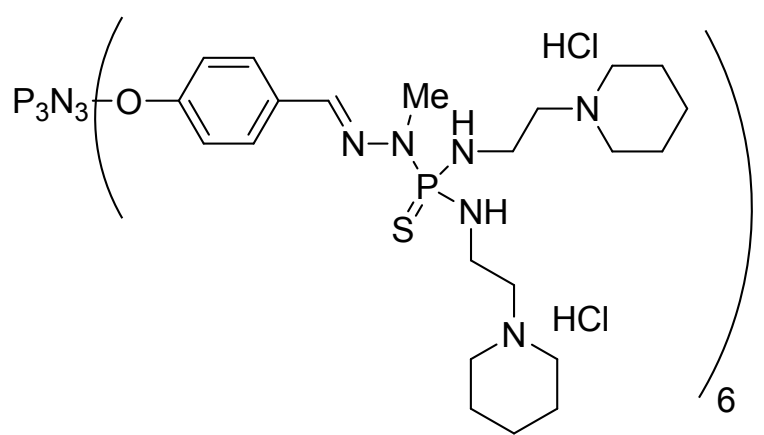

Molecular formula: $\mathrm{C}_{132} \mathrm{H}_{240} \mathrm{Cl}_{12} \mathrm{~N}_{39} \mathrm{O}_{6} \mathrm{P}_{9} \mathrm{~S}_{6}$; MW: 3366. ${ }^{1} \mathrm{H}$ NMR $\left(400 \mathrm{MHz}, \mathrm{D}_{2} \mathrm{O}\right) \delta=7.73(\mathrm{~s}, 6 \mathrm{H}), 7.58(\mathrm{~d}, \mathrm{~J}=8.0 \mathrm{~Hz}, 12 \mathrm{H}), 6.88(\mathrm{~d}, \mathrm{~J}$ $=8.0 \mathrm{~Hz}, 12 \mathrm{H}), 3.42(\mathrm{~m}, 24 \mathrm{H}), 3.30(\mathrm{~m} \mathrm{24H}), 3.15(\mathrm{~m}, 42 \mathrm{H}), 2.82(\mathrm{~m}, 24 \mathrm{H}), 1.74(\mathrm{~m}, 24 \mathrm{H}), 1.61(\mathrm{~m}, 36 \mathrm{H}), 1.32(\mathrm{~m}, 12 \mathrm{H}) .{ }^{31} \mathrm{P}\left\{{ }^{1} \mathrm{H}\right\}$ $\operatorname{NMR}\left(161 \mathrm{MHz}, \mathrm{D}_{2} \mathrm{O}\right) \delta=69.73,9.20 .{ }^{13} \mathrm{C}\left\{{ }^{1} \mathrm{H}\right\} \operatorname{NMR}\left(100 \mathrm{MHz}, \mathrm{D}_{2} \mathrm{O}\right) \delta=149.91(\mathrm{~d}, \mathrm{~J}=4 \mathrm{~Hz}), 139.37(\mathrm{~d}, \mathrm{~J}=14 \mathrm{~Hz}), 133.15,128.27$, 121.32, 57.00 (d, J = $7 \mathrm{~Hz}), 53.59,35.76,31.93$ (d, J = $10 \mathrm{~Hz}), 22.62,20.93$.

\section{Compound 7G1}

$\{[\{N, N$-(2-(piperidin-1-yl)ethanamine $\}$-phosphonothioate $]-\{4-((2-m e t h y l h y d r a z o n o) m e t h y l)\}$ phenoxy $\}-1,3,5,2,4,6-$ triazatriphosphorine 


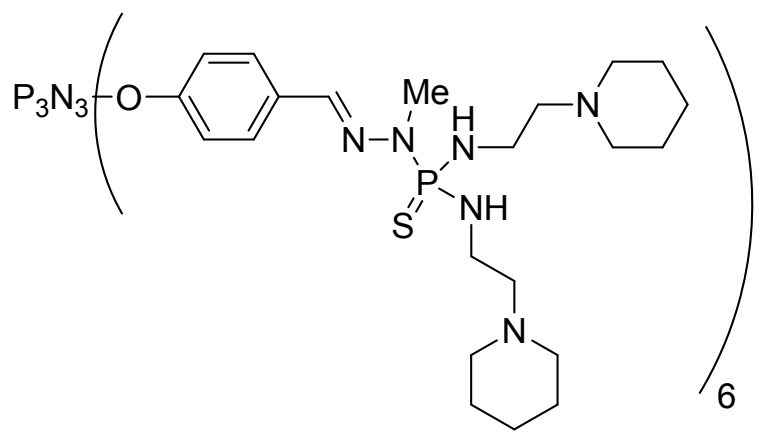

Molecular formula: $\mathrm{C}_{132} \mathrm{H}_{228} \mathrm{~N}_{39} \mathrm{O}_{6} \mathrm{P}_{9} \mathrm{~S}_{6}$; MW: 2929. ${ }^{1} \mathrm{H}$ NMR (400 MHz, CDCl3) $\delta=7.51(\mathrm{~s}, 6 \mathrm{H}), 7.46(\mathrm{~d}, \mathrm{~J}=9.0 \mathrm{~Hz}, 12 \mathrm{H}), 6.93(\mathrm{~d}, \mathrm{~J}=$ $9.0 \mathrm{~Hz}, 12 \mathrm{H}), 4.11-4.04(\mathrm{~m}, 12 \mathrm{H}), 3.16(\mathrm{~d}, \mathrm{~J}=12 \mathrm{~Hz}, 18 \mathrm{H}), 3.12-2.94(\mathrm{~m}, 24 \mathrm{H}), 2.43(\mathrm{t}, \mathrm{J}=3 \mathrm{~Hz}, 24 \mathrm{H}), 2.35(\mathrm{brs}, 48 \mathrm{H}), 1.53(\mathrm{t}, \mathrm{J}=3$ $\mathrm{Hz}, 48 \mathrm{H}), 1.39$ (brs, 24H). (Yield = 76\%); ${ }^{31} \mathrm{P}\left\{{ }^{1} \mathrm{H}\right\} \mathrm{NMR}(121 \mathrm{MHz}, \mathrm{CDCl} 3) \delta=68.55,8.24 ;\left(100 \mathrm{MHz}, \mathrm{CDCl}_{3}\right) \delta 150.6,135.9,132.8$, 127.5, 121.0, 58.9, 54.3, 37.8, 30.8, 25.8, 24.3. ${ }^{13} \mathrm{C}\left\{{ }^{1} \mathrm{H}\right\} \mathrm{NMR}\left(75 \mathrm{MHz}, \mathrm{CDCl}_{3}\right) \delta 150.64$ (d, J = $\left.4 \mathrm{~Hz}\right), 135.85$ (d, J = $\left.16 \mathrm{~Hz}\right), 132.84$, 127.52, 121.02, 58.92 (d, J = $11 \mathrm{~Hz}), 54.35,37.86,30.73$ (d, J = $13 \mathrm{~Hz}), 25.98,24.39$. MS (ES+): Cal. Mass 2929.5; Obs. Mass $2929.6[\mathrm{M}]^{+}$

\section{Compound 8G1, $\mathrm{HCl}$}

$\{[\{N, N$-(3-(pyrrolidin-1-yl)propanamine $\}$-phosphonothioate $]-\{4-((2-m e t h y l h y d r a z o n o) m e t h y l)\}$ phenoxy $\}-1,3,5,2,4,6-$ triazatriphosphorine, dodeca (12) hydrochloride

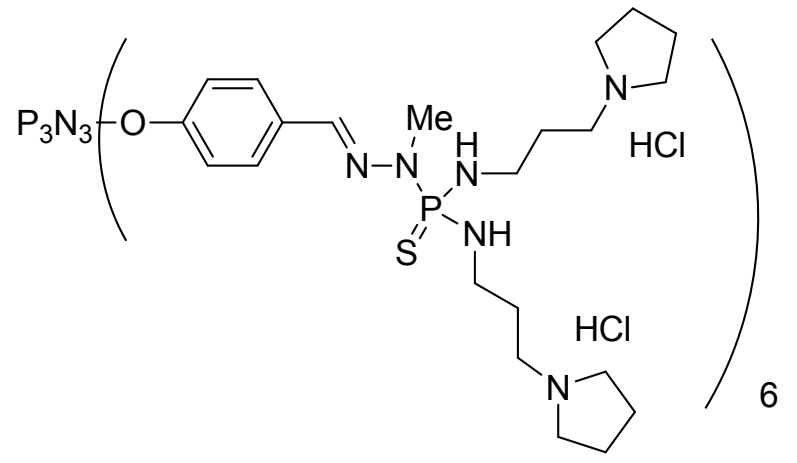


Molecular formula: $\mathrm{C}_{132} \mathrm{H}_{240} \mathrm{Cl}_{12} \mathrm{~N}_{39} \mathrm{O}_{6} \mathrm{P}_{9} \mathrm{~S}_{6}, \mathrm{MW}: 3366$.

\section{Compound 8G1}

$\{[\{N, N$-(3-(pyrrolidin-1-yl)propanamine $\}$-phosphonothioate $]-\{4-((2-m e t h y l h y d r a z o n o) m e t h y l)\}$ phenoxy $\}-1,3,5,2,4,6-$ triazatriphosphorine

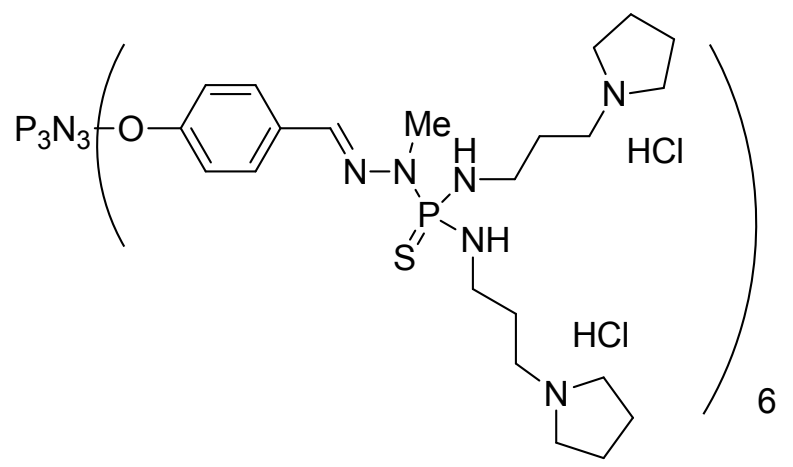

Molecular formula: $\mathrm{C}_{132} \mathrm{H}_{228} \mathrm{~N}_{39} \mathrm{O}_{6} \mathrm{P}_{9} \mathrm{~S}_{6}, \mathrm{MW}: 2929 .{ }^{1} \mathrm{H}$ NMR $\left(300 \mathrm{MHz}, \mathrm{CDCl}_{3}\right) \delta=7.48(\mathrm{~s}, 6 \mathrm{H}), 7.46(\mathrm{br} d, \mathrm{~J}=8 \mathrm{~Hz}, 12 \mathrm{H}), 6.99(\mathrm{~d}, \mathrm{~J}=$ $8 \mathrm{~Hz}, 12 \mathrm{H}), 4.09(\mathrm{~m}, 12 \mathrm{H}), 3.19(\mathrm{~d}, \mathrm{~J}=10 \mathrm{~Hz}, 18 \mathrm{H}), 3.10(\mathrm{~m}, 24 \mathrm{H}), 2.54(\mathrm{~m}, 24 \mathrm{H}), 2.47(\mathrm{br} \mathrm{m}, 48 \mathrm{H}), 1.72(\mathrm{br} \mathrm{m}, 72 \mathrm{H}) .{ }^{31} \mathrm{P}\left\{{ }^{1} \mathrm{H}\right\} \mathrm{NMR}$ $\left(121 \mathrm{MHz}, \mathrm{CDCl}_{3}\right) \delta=67.22,8.18 .{ }^{13} \mathrm{C}\left\{{ }^{1} \mathrm{H}\right\} \mathrm{NMR}\left(100 \mathrm{MHz}, \mathrm{CDCl}_{3}\right) \delta=150.58(\mathrm{~d}, \mathrm{~J}=6 \mathrm{~Hz}), 135.72(\mathrm{~d}, \mathrm{~J}=13 \mathrm{~Hz}), 132.92,127.41$, 121.04, 54.62, 54.15, 40.72, 31.27 (d, J = 12Hz), 29.78 (d, J = $8 \mathrm{~Hz}), 23.45$.

\section{Compound 9G1,HCl}

$\{[\{N, N-(3-($ piperidin-1-yl)propanamine $\}$-phosphonothioate $]-\{4-((2-m e t h y l h y d r a z o n o) m e t h y l)\}$ phenoxy $\}-1,3,5,2,4,6-$ triazatriphosphorine, dodeca (12) hydrochloride 


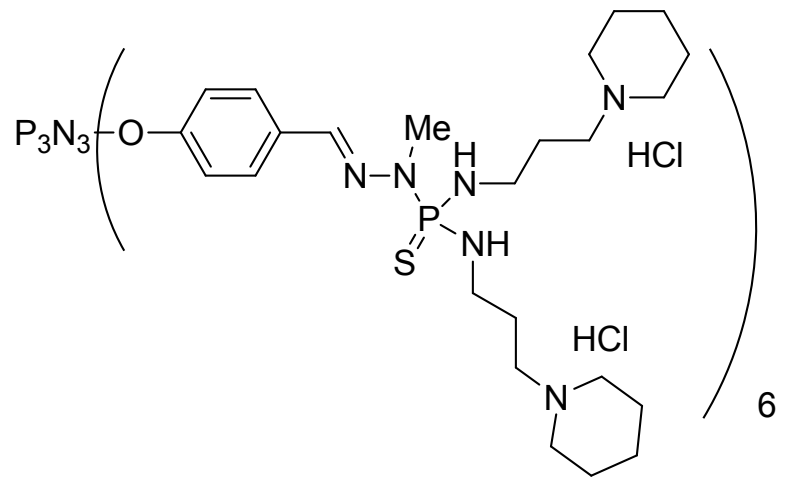

Molecular formula: $\mathrm{C}_{144} \mathrm{H}_{264} \mathrm{Cl}_{12} \mathrm{~N}_{39} \mathrm{O}_{6} \mathrm{P}_{9} \mathrm{~S}_{6} ; \mathrm{MW}: 3534$.

\section{Compound 9G1}

$\{[\{N, N-(3-($ piperidin-1-yl)propanamine $\}-$ phosphonothioate $]-\{4-((2-m e t h y l h y d r a z o n o) m e t h y l)\}$ phenoxy $\}-1,3,5,2,4,6-$ triazatriphosphorine

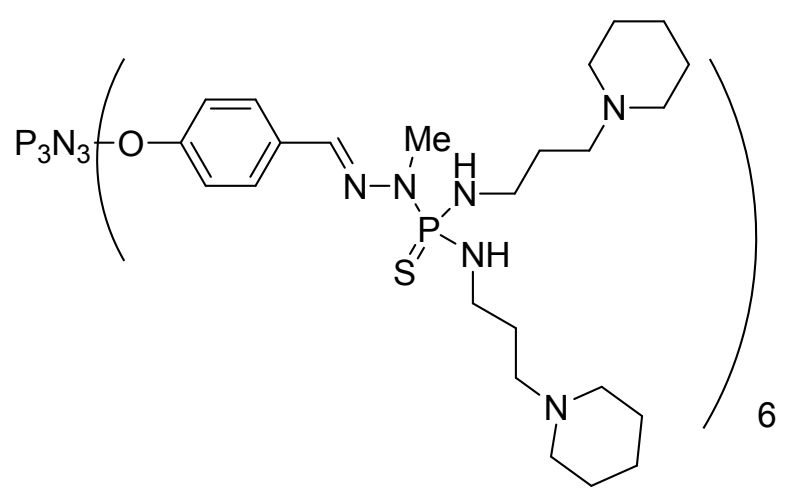

Molecular formula: $\mathrm{C}_{144} \mathrm{H}_{252} \mathrm{~N}_{39} \mathrm{O}_{6} \mathrm{P}_{9} \mathrm{~S}_{6}$; MW: 3097. ${ }^{1} \mathrm{H}$ NMR $(300 \mathrm{MHz}, \mathrm{CDCl} 3) \delta=7.49(\mathrm{~s}, 6 \mathrm{H}), 7.46(\mathrm{~d}, \mathrm{~J}=9.0 \mathrm{~Hz}, 12 \mathrm{H}), 6.98(\mathrm{~d}, \mathrm{~J}=$ $9.0 \mathrm{~Hz}, 12 \mathrm{H}), 4.36-4.29(\mathrm{~m}, 12 \mathrm{H}), 3.20(\mathrm{~d}, \mathrm{~J}=12 \mathrm{~Hz}, 18 \mathrm{H}), 3.11-3.00(\mathrm{~m}, 24 \mathrm{H}), 2.42-2.38(\mathrm{~m}, 72 \mathrm{H}), 1.74-1.66(\mathrm{~m}, 24 \mathrm{H}), 1.57-1.53$ $(\mathrm{m}, 48 \mathrm{H}), 1.40-1.39(\mathrm{~m}, 24 \mathrm{H}) .($ Yield $=75.4 \%) ;{ }^{31} \mathrm{P}\left\{{ }^{1} \mathrm{H}\right\} \mathrm{NMR}(121 \mathrm{MHz}, \mathrm{CDCl} 3) \delta=67.03,8.19 ;{ }^{13} \mathrm{C}\left\{{ }^{1} \mathrm{H}\right\} \mathrm{NMR}(75 \mathrm{MHz}, \mathrm{CDCl} 3) \delta=$ 
$150.55(\mathrm{~d}, \mathrm{~J}=5 \mathrm{~Hz}), 135.70(\mathrm{~d}, \mathrm{~J}=12 \mathrm{~Hz}), 132.98,127.43,121.02,57.88,54.64,41.15,31.42(\mathrm{~d}, \mathrm{~J}=11 \mathrm{~Hz}), 27.41(\mathrm{~d}, \mathrm{~J}=8 \mathrm{~Hz})$, 25.95, 24.39.

\section{Compound 10G1,, $\mathrm{HCl}$}

$\{[\{N, N$-(3-(morpholino-1-yl)propanamine $\}$-phosphonothioate]-\{4-((2-methylhydrazono)methyl) $\}$ phenoxy $\}-1,3,5,2,4,6-$ triazatriphosphorine, dodeca (12) hydrochloride

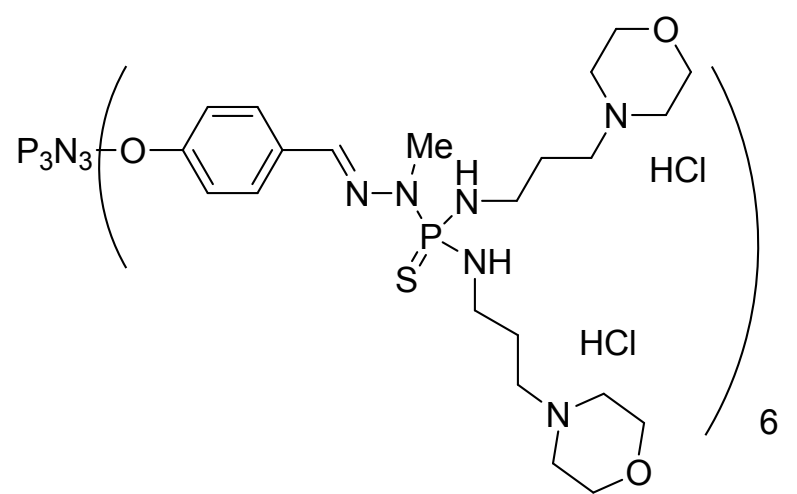

Molecular formula: $\mathrm{C}_{132} \mathrm{H}_{240} \mathrm{Cl}_{12} \mathrm{~N}_{39} \mathrm{O}_{18} \mathrm{P}_{9} \mathrm{~S}_{6}, \mathrm{MW}: 3558$.

\section{Compound 10G1}

$\{[\{N, N$-(3-(morpholino-1-yl)propanamine $\}$-phosphonothioate $]-\{4-((2-m e t h y l h y d r a z o n o) m e t h y l)\}$ phenoxy $\}-1,3,5,2,4,6-$ triazatriphosphorine

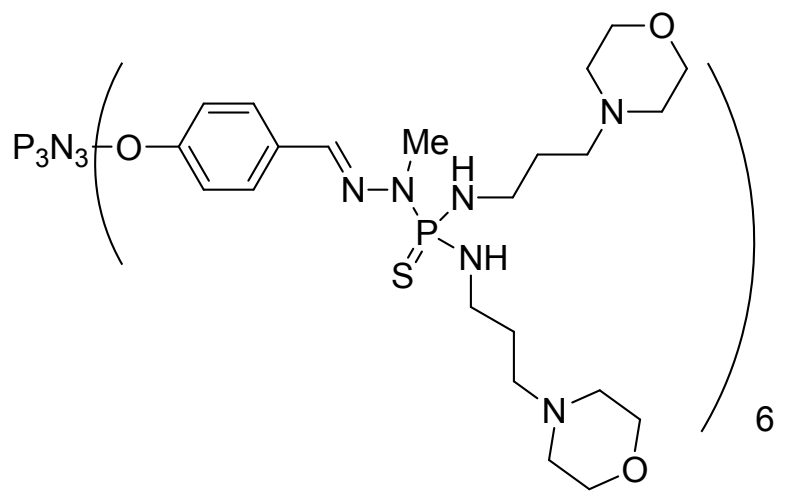


Molecular formula: $\mathrm{C}_{132} \mathrm{H}_{228} \mathrm{~N}_{39} \mathrm{O}_{18} \mathrm{P}_{9} \mathrm{~S}_{6}$; MW: 3121. ${ }^{1} \mathrm{H}$ NMR $(400 \mathrm{MHz}, \mathrm{CDCl} 3) \delta=7.50(\mathrm{~s}, 6 \mathrm{H}), 7.45(\mathrm{~d}, \mathrm{~J}=9 \mathrm{~Hz}, 12 \mathrm{H}), 6.99(\mathrm{~d}, \mathrm{~J}=$ $9 \mathrm{~Hz}, 12 \mathrm{H}$ ), 4.11 (brs, 12H), 3.67 (t, J = $8 \mathrm{~Hz}, 48 \mathrm{H}), 3.20$ (d, J = $12 \mathrm{~Hz}, 18 \mathrm{H}), 3.07$ (brs, 24H), 2.46-2.43 (m, 72H), $1.74-1.68(\mathrm{~m}$, 24H). (Yield = 79.4\%); ${ }^{31} \mathrm{P}\left\{{ }^{1} \mathrm{H}\right\}$ NMR $(161 \mathrm{MHz}, \mathrm{CDCl} 3) \delta=67.22,8.14 ;{ }^{13} \mathrm{C}\left\{{ }^{1} \mathrm{H}\right\} \mathrm{NMR}(100 \mathrm{MHz}, \mathrm{CDCl} 3) \delta=150.62(\mathrm{~d}, \mathrm{~J}=8 \mathrm{~Hz})$ 135.91 (d, J = $13 \mathrm{~Hz}), 132.89,127.44,121.08,66.86,57.34,53.70,40.77,31.40$ (d, J = $12 \mathrm{~Hz}), 27.19$ (d, J = $7 \mathrm{~Hz})$

\section{compound $11 \mathrm{G} 1_{, \mathrm{HCl}}$}

$\{[\{N, N$-(3-(1-methypyrrolidino-2-yl)ethanamine $\}$-phosphonothioate $]-\{4-((2-m e t h y l h y d r a z o n o) m e t h y l)\}$ phenoxy $\}-1,3,5,2,4,6-$ triazatriphosphorine, dodeca (12) hydrochloride

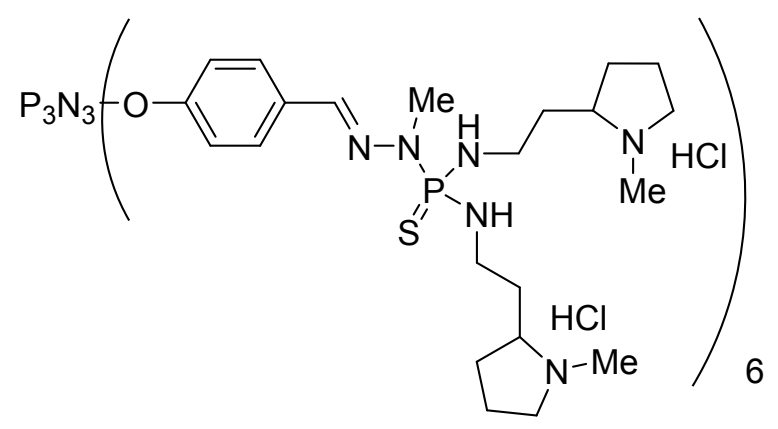

Molecular formula: $\mathrm{C}_{132} \mathrm{H}_{240} \mathrm{Cl}_{12} \mathrm{~N}_{39} \mathrm{O}_{6} \mathrm{P}_{9} \mathrm{~S}_{6}, \mathrm{MW}: 3366$.

\section{compound 11G1}

$\{[\{N, N$-(3-(1-methypyrrolidino-2-yl)ethanamine $\}$-phosphonothioate $]-\{4-((2-m e t h y l h y d r a z o n o) m e t h y l)\}$ phenoxy $\}-1,3,5,2,4,6-$ triazatriphosphorine 


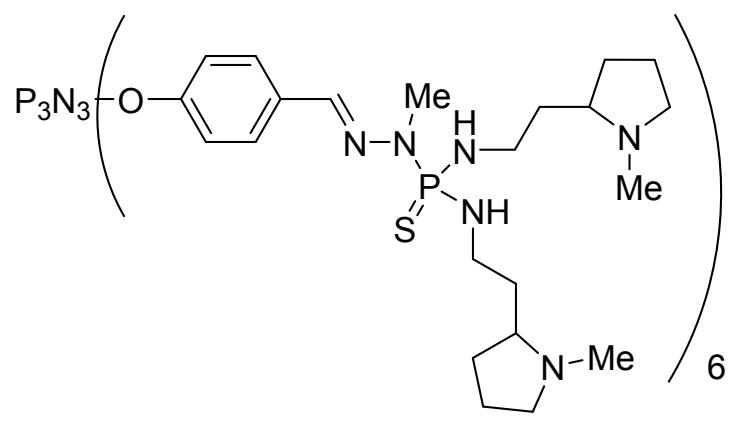

Molecular formula: $\mathrm{C}_{132} \mathrm{H}_{228} \mathrm{~N}_{39} \mathrm{O}_{6} \mathrm{P}_{9} \mathrm{~S}_{6}, \mathrm{MW}: 2929 .{ }^{1} \mathrm{H}$ NMR $(300 \mathrm{MHz}, \mathrm{CDCl} 3) \delta=7.48(\mathrm{~s}, 6 \mathrm{H}), 7.45(\mathrm{~d}, \mathrm{~J}=6 \mathrm{~Hz}, 12 \mathrm{H}), 6.98(\mathrm{~d}, \mathrm{~J}=6$ $\mathrm{Hz}, 12 \mathrm{H}), 3.94-3.79(\mathrm{~m}, 12 \mathrm{H}), 3.16(\mathrm{~d}, \mathrm{~J}=12 \mathrm{~Hz}, 18 \mathrm{H}), 3.09-2.99(\mathrm{~m}, 36 \mathrm{H}), 2.28(\mathrm{~s}, 36 \mathrm{H}), 2.17-2.05(\mathrm{~m}, 24 \mathrm{H}), 1.96-1.45(\mathrm{~m}, 72 \mathrm{H})$. (Yield = 78\%); ${ }^{11} \mathrm{P}\left\{{ }^{1} \mathrm{H}\right\}$ NMR $(121 \mathrm{MHz}, \mathrm{CDCl} 3) \delta=68.94,7.84 ;{ }^{13} \mathrm{C}\left\{{ }^{1} \mathrm{H}\right\} \mathrm{NMR}(75 \mathrm{MHz}, \mathrm{CDCl} 3) \delta=150.65,135.97(\mathrm{~d}, \mathrm{~J}=13 \mathrm{~Hz})$, $132.80,127.46,121.05,64.44,64.40,57.15,40.60,38.72,38.68,34.16 ; 34.17,33.99,33.90,30.98$ (d, J = 12 Hz), $30.03,30.00$, $29.95,22.14$.

Preparation of compounds of generation 1 (5G1, 13G1, 14G1, 15G1, 17G1, 18G1, 19G1 and 20G1) and (5G1,, HCl, 13G1,, HCl, 14G1,, HCl, 15G1,, HCl, 17G1,, HCl, 18G1,, HCl, 19G1,, HCl and 20G1,, HCl) (Scheme S4)

The dendrimer 8 was condensed with the diamines 7a,b,e,f, h, i, j, k, 1 (THF, room temperature, overnight) to afford 9a,b,e,f, h, i, j, k, 1 which were further converted to their respective phosphorus dendrimers bearing the amines 5G1, 13G1, 14G1, 15G1, 17G1, 18G1, 19G1 and 20G1 by action of sodium borohydride, in THF-Methanol mixture, at room temperature overnight (yields 76 to $91 \%$ ). Then, $5 \mathrm{G} 1,13 \mathrm{G} 1,14 \mathrm{G} 1,15 \mathrm{G} 1,17 \mathrm{G} 1,18 \mathrm{G} 1,19 \mathrm{G} 1$ and $20 \mathrm{G} 1$ were further converted to their respective cationic dendrimers $5 \mathrm{G} 1_{\text {,HCl}}$, $13 \mathrm{G}_{, \mathrm{HCl}}, 14 \mathrm{G} 1_{, \mathrm{HCl}}, 15 \mathrm{G}_{, \mathrm{HCl}}, 17 \mathrm{G} 1_{, \mathrm{HCl}}, 18 \mathrm{G} 1_{, \mathrm{HCl}}, 19 \mathrm{G} 1_{, \mathrm{HCl}}$, and $20 \mathrm{G} 1_{, \mathrm{HCl}}$ ) by protonation with hydrogen chloride (in diethylether), in $\mathrm{THF}$ at $0^{\circ} \mathrm{C}$, reaching slowly room temperature overnight (quantitative yield) 

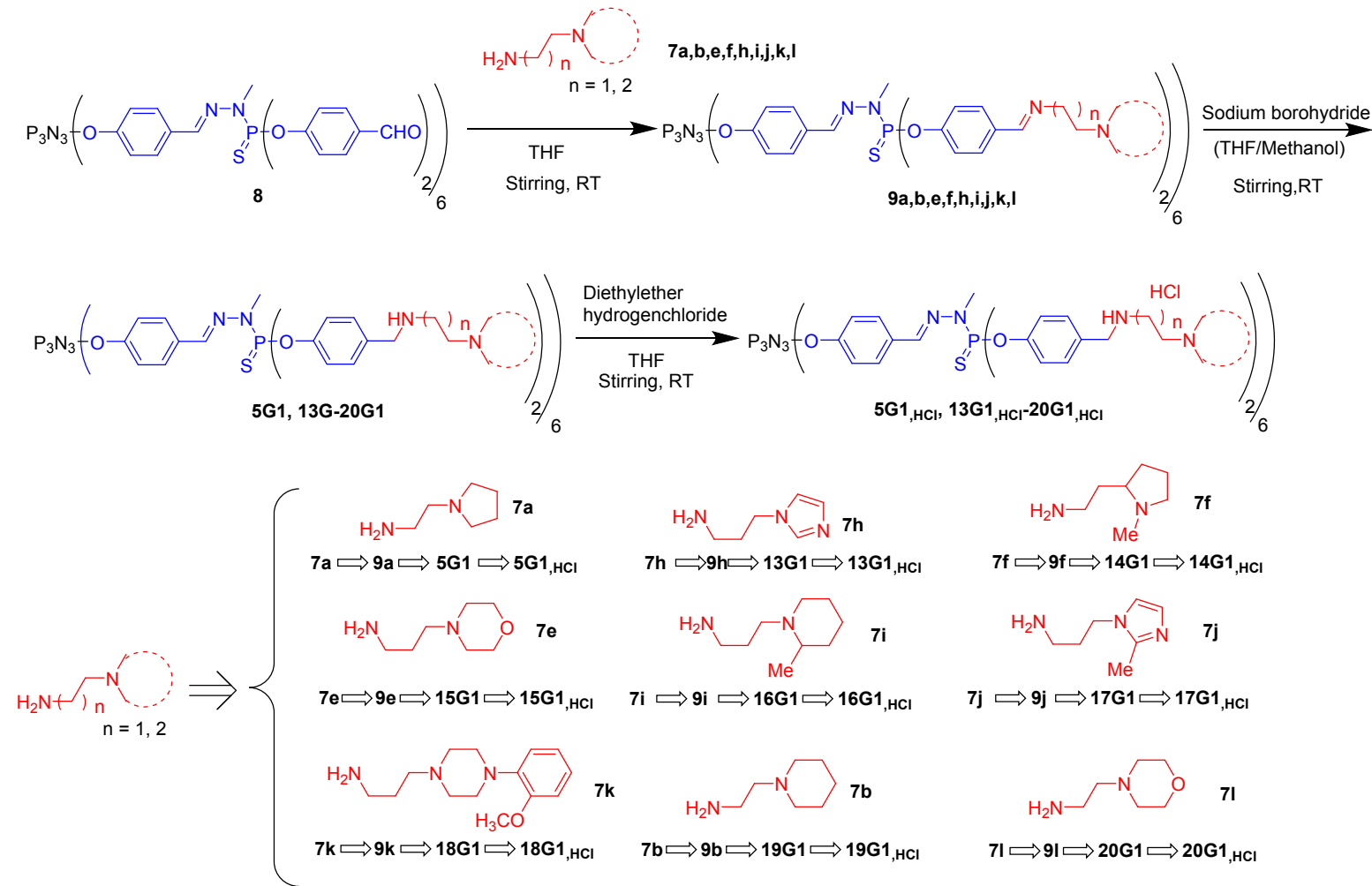

\section{Scheme S4}

The compound 8 has been prepared according to the procedure described in the publications [a) Slany M., Bardají M., Casanove M.J., Caminade A.M., Majoral J.P., Chaudret B. Dendrimer surface chemistry. An easy access to polyphosphines and their gold complexes. J. Am. Chem. Soc. 1995, 117, 9764; b) Launay N., Caminade A.M., Majoral J.P. Synthesis of bowl-shaped dendrimers from generation 1 to generation 8. J. Organomet. Chem. 1997, 529, 51]

\section{Compound 5G1, $\mathrm{HCl}$}

$\{[\{O, O-4-(((2-($ pyrrolidin-1-yl)ethyl)amino)methyl)phenoxy $\}$-phosphonothioate $]-\{4-((2-m e t h y l h y d r a z o n o) m e t h y l)\}$ phenoxy $\}-$ 1,3,5,2,4,6-triazatriphosphorine, tetracosa (24) hydrochloride 


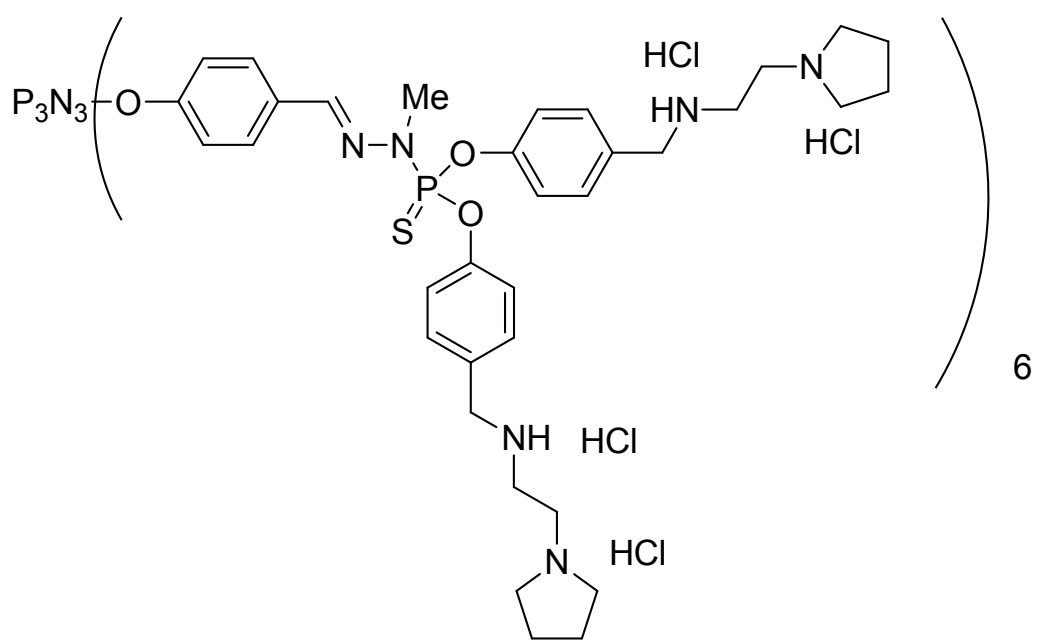

Molecular formula: $\mathrm{C}_{204} \mathrm{H}_{300} \mathrm{Cl}_{24} \mathrm{~N}_{39} \mathrm{O}_{18} \mathrm{P}_{9} \mathrm{~S}_{6}, \mathrm{MW}: 4909$.

\section{Compound \\ 5G1: \\ $\{[\{O, O-4-(((2-($ pyrrolidin-1-yl)ethyl)amino)methyl $)$ phenoxy $\}$-phosphonothioate $]-\{4-((2-$} methylhydrazono)methyl)\}phenoxy $\}$-1,3,5,2,4,6-triazatriphosphorine

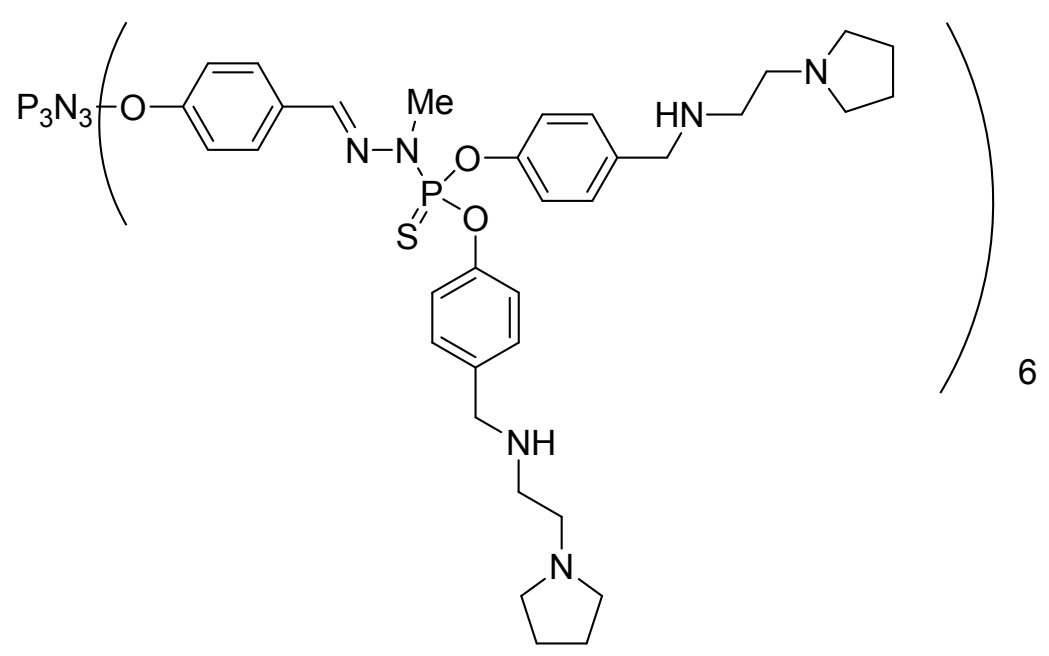


Molecular formula: $\mathrm{C}_{204} \mathrm{H}_{276} \mathrm{~N}_{39} \mathrm{O}_{18} \mathrm{P}_{9} \mathrm{~S}_{6}, \mathrm{MW}: 4034 .{ }^{1} \mathrm{H}$ NMR $(400 \mathrm{MHz}, \mathrm{CDCl} 3) \delta=7.62(\mathrm{~d}, \mathrm{~J}=8 \mathrm{~Hz}, 12 \mathrm{H}), 7.60(\mathrm{~s}, 6 \mathrm{H}), 7.23(\mathrm{~d}, \mathrm{~J}=$ $8 \mathrm{~Hz}, 24 \mathrm{H}), 7.13(\mathrm{~d}, \mathrm{~J}=8 \mathrm{~Hz}, 24 \mathrm{H}), 7.01(\mathrm{~d}, \mathrm{~J}=8 \mathrm{~Hz}, 12 \mathrm{H}), 3.73(\mathrm{~s}, 24 \mathrm{H}), 3.25(\mathrm{~d}, \mathrm{~J}=12 \mathrm{~Hz}, 18 \mathrm{H}), 2.71(\mathrm{t}, \mathrm{J}=4 \mathrm{~Hz}, 24 \mathrm{H}), 2.60(\mathrm{t}, \mathrm{J}$ $=8 \mathrm{~Hz}, 24 \mathrm{H}), 2.49$ (brs, 48H), $1.75(\mathrm{~s}, 48 \mathrm{H})$. (Yield = 91\%); ${ }^{31} \mathrm{P}\left\{{ }^{1} \mathrm{H}\right\} \mathrm{NMR}(121 \mathrm{MHz}, \mathrm{CDCl} 3) \delta=62.92(\mathrm{~s}), 8.39(\mathrm{~s})$.

\section{Compound 19G1,, $\mathrm{HCl}$}

$\{[\{O, O-4-(((2-($ piperidin-1-yl)ethyl)amino)methyl)phenoxy $\}$-phosphonothioate $]-\{4-((2-$ methylhydrazono $)$ methyl $)\}$ phenoxy $\}-$ 1,3,5,2,4,6-triazatriphosphorine, tetracosa (24) hydrochloride

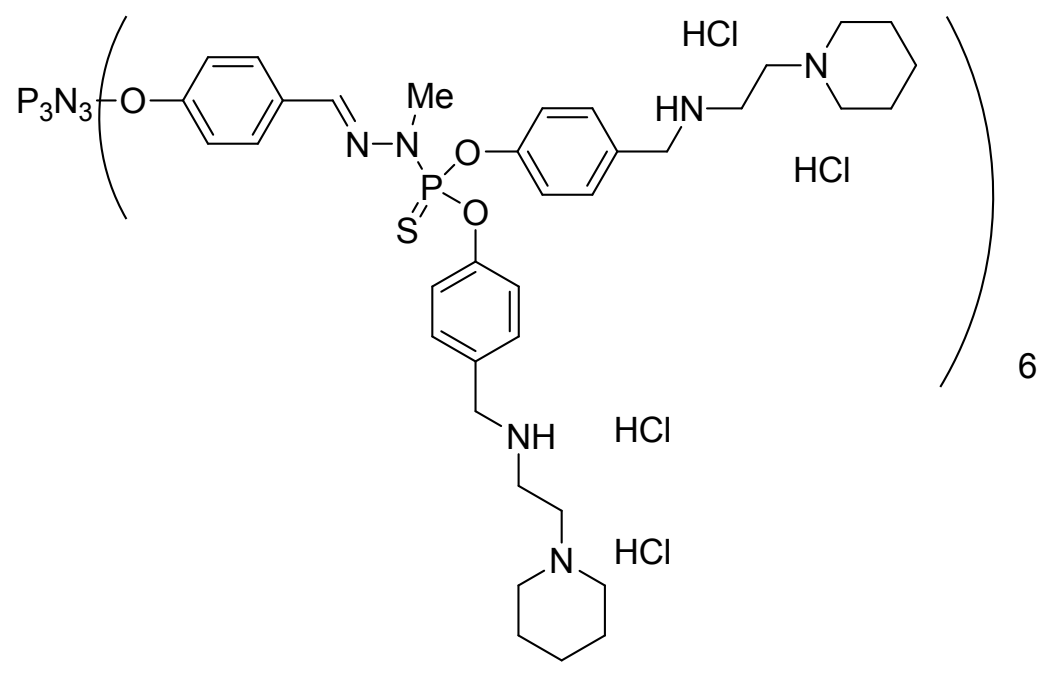

Molecular formula: $\mathrm{C}_{216} \mathrm{H}_{324} \mathrm{Cl}_{24} \mathrm{~N}_{39} \mathrm{O}_{18} \mathrm{P}_{9} \mathrm{~S}_{6}, \mathrm{MW}: 5077$.

\section{Compound 19G1}

$\{[\{O, O-4-(((2-($ piperidin-1-yl)ethyl)amino)methyl)phenoxy $\}$-phosphonothioate $]-\{4-((2-$ methylhydrazono $)$ methyl $)\}$ phenoxy $\}-$ 1,3,5,2,4,6-triazatriphosphorine 


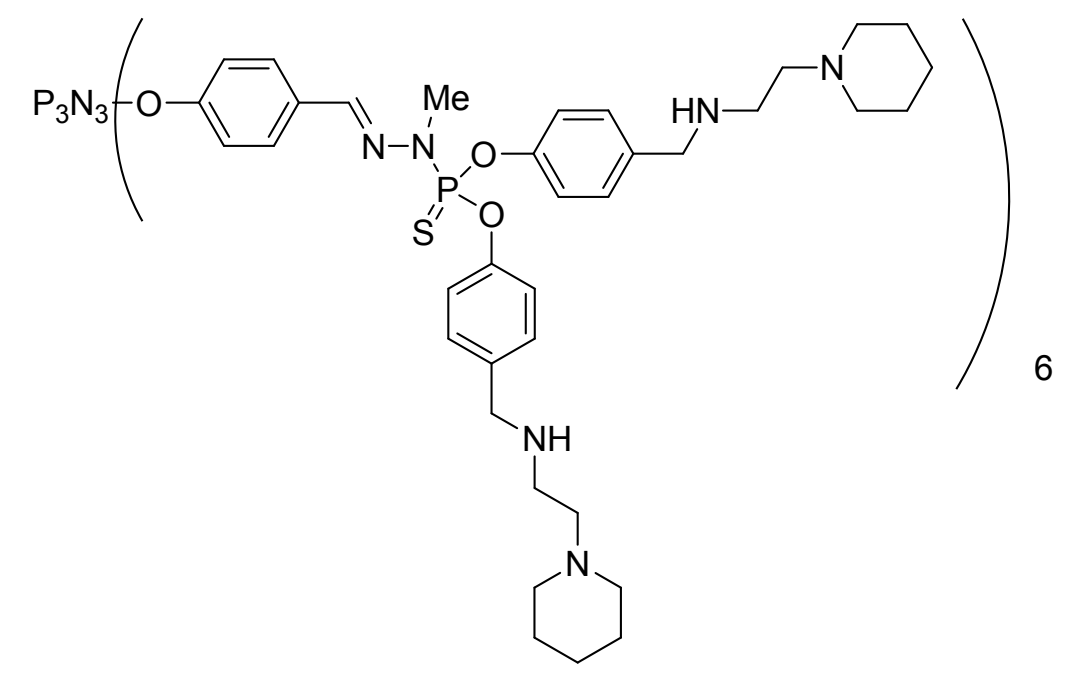

Molecular formula: $\mathrm{C}_{216} \mathrm{H}_{300} \mathrm{~N}_{39} \mathrm{O}_{18} \mathrm{P}_{9} \mathrm{~S}_{6}, \mathrm{MW}: 4202 .{ }^{1} \mathrm{H}$ NMR $(400 \mathrm{MHz}, \mathrm{CDCl} 3) \delta=7.62(\mathrm{~d}, \mathrm{~J}=8 \mathrm{~Hz}, 12 \mathrm{H}), 7.59(\mathrm{~s}, 6 \mathrm{H}), 7.24-7.20$ $(\mathrm{m}, 24 \mathrm{H}), 7.13(\mathrm{~d}, \mathrm{~J}=8 \mathrm{~Hz}, 24 \mathrm{H}), 7.01(\mathrm{~d}, \mathrm{~J}=8 \mathrm{~Hz}, 12 \mathrm{H}), 3.72(\mathrm{~s}, 24 \mathrm{H}), 3.24(\mathrm{~d}, \mathrm{~J}=12 \mathrm{~Hz}, 18 \mathrm{H}), 2.66(\mathrm{t}, \mathrm{J}=8 \mathrm{~Hz}, 24 \mathrm{H}), 2.41(\mathrm{t}, \mathrm{J}=$ $8 \mathrm{~Hz}, 24 \mathrm{H}), 2.32$ (brs, 48H), 1.55-1.52 (m, 48H), 1.29 (brs, 24H). (Yield = 90\%); ${ }^{31} \mathrm{P}\left\{{ }^{1} \mathrm{H}\right\} \mathrm{NMR}(161 \mathrm{MHz}, \mathrm{CDCl} 3) \delta=62.63(\mathrm{~s}), 8.37$ (s); ${ }^{13} \mathrm{C}\left\{{ }^{1} \mathrm{H}\right\} \operatorname{NMR}(100 \mathrm{MHz}, \mathrm{CDCl} 3) \delta=151.20,149.41$ (d, $\left.\mathrm{J}=7 \mathrm{~Hz}\right), 138.10$ (brs), 137.84, 132.21, 129.15, 128.24, 121.30, 121.16, 58.54, 54.69, 53.32, 46.02, 33.01 (d, J = 14 Hz), 26.02, 24.45.

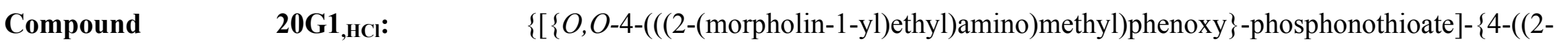
methylhydrazono)methyl) $\}$ phenoxy $\}-1,3,5,2,4,6$-triazatriphosphorine, tetracosa (24 hydrochloride 


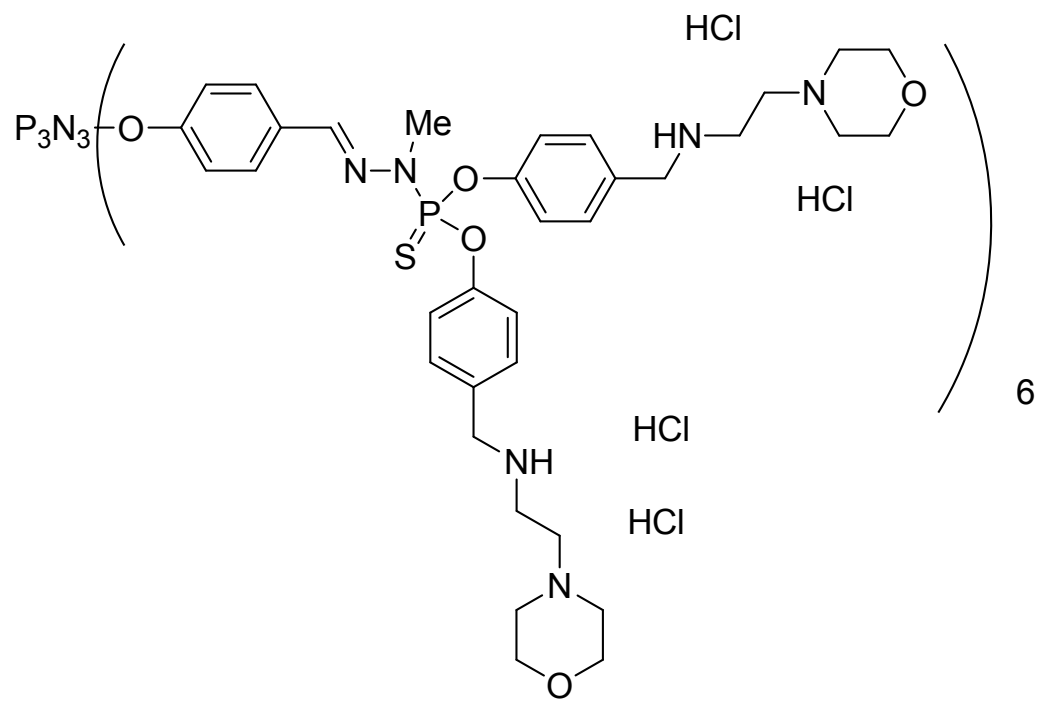

Molecular formula: $\mathrm{C}_{204} \mathrm{H}_{300} \mathrm{Cl}_{24} \mathrm{~N}_{39} \mathrm{O}_{30} \mathrm{P}_{9} \mathrm{~S}_{6}$, MW: 5101.

\section{Compound 20G1}

$\{[\{O, O-4-(((2-($ morpholin-1-yl)ethyl)amino)methyl)phenoxy $\}$-phosphonothioate $]-\{4-((2-m e t h y l h y d r a z o n o) m e t h y l)\}$ phenoxy $\}-$ 1,3,5,2,4,6-triazatriphosphorine 


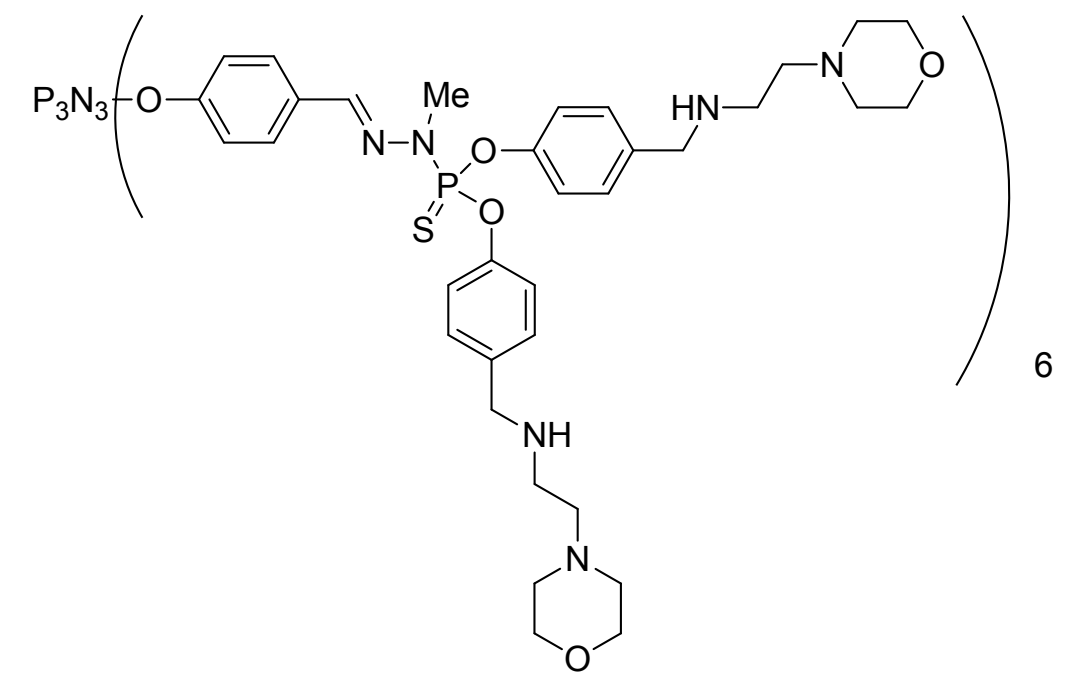

Molecular formula: $\mathrm{C}_{204} \mathrm{H}_{276} \mathrm{~N}_{39} \mathrm{O}_{30} \mathrm{P}_{9} \mathrm{~S}_{6}, \mathrm{MW}: 4225 .{ }^{1} \mathrm{H}$ NMR $(300 \mathrm{MHz}, \mathrm{CDCl} 3) \delta=7.63(\mathrm{~d}, \mathrm{~J}=8 \mathrm{~Hz}, 12 \mathrm{H}), 7.61(\mathrm{~s}, 6 \mathrm{H}), 7.23(\mathrm{~d}, \mathrm{~J}=8$ $\mathrm{Hz}, 24 \mathrm{H}), 7.13(\mathrm{~d}, \mathrm{~J}=8 \mathrm{~Hz}, 24 \mathrm{H}), 7.01(\mathrm{~d}, \mathrm{~J}=8 \mathrm{~Hz}, 12 \mathrm{H}), 3.73(\mathrm{~s}, 24 \mathrm{H}), 3.67(\mathrm{t}, \mathrm{J}=6 \mathrm{~Hz}, 48 \mathrm{H}), 3.25(\mathrm{~d}, \mathrm{~J}=12 \mathrm{~Hz}, 18 \mathrm{H}), 2.66(\mathrm{t}, \mathrm{J}=$ $8 \mathrm{~Hz}, 24 \mathrm{H}), 2.40-2.37(\mathrm{~m}, 48 \mathrm{H})$. (Yield = 87.4\%); ${ }^{31} \mathrm{P}\left\{{ }^{1} \mathrm{H}\right\} \mathrm{NMR}(121 \mathrm{MHz}, \mathrm{CDCl} 3) \delta=62.95(\mathrm{~s}), 8.39(\mathrm{~s}) ;{ }^{13} \mathrm{C}\left\{{ }^{1} \mathrm{H}\right\} \mathrm{NMR}(75 \mathrm{MHz}$, $\mathrm{CDCl} 3) \delta=151.19,149.48(\mathrm{~d}, \mathrm{~J}=9 \mathrm{~Hz}), 138.50$ (d, J = $19 \mathrm{~Hz}), 137.70,132.22,129.19,128.26,122.09,121.26,66.97,58.21,53.69$, 53.27, 45.37, $32.99(\mathrm{~d}, \mathrm{~J}=15 \mathrm{~Hz})$.

\section{Compound 13G1, $\mathrm{HCl}$}

$\{[\{O, O-4-(((2-($ imidazoly-1-yl)propyl)amino)methyl)phenoxy $\}$-phosphonothioate $]-\{4-((2-m e t h y l h y d r a z o n o) m e t h y l)\}$ phenoxy $\}-$ 1,3,5,2,4,6-triazatriphosphorine, tetracosa (24) hydrochloride 


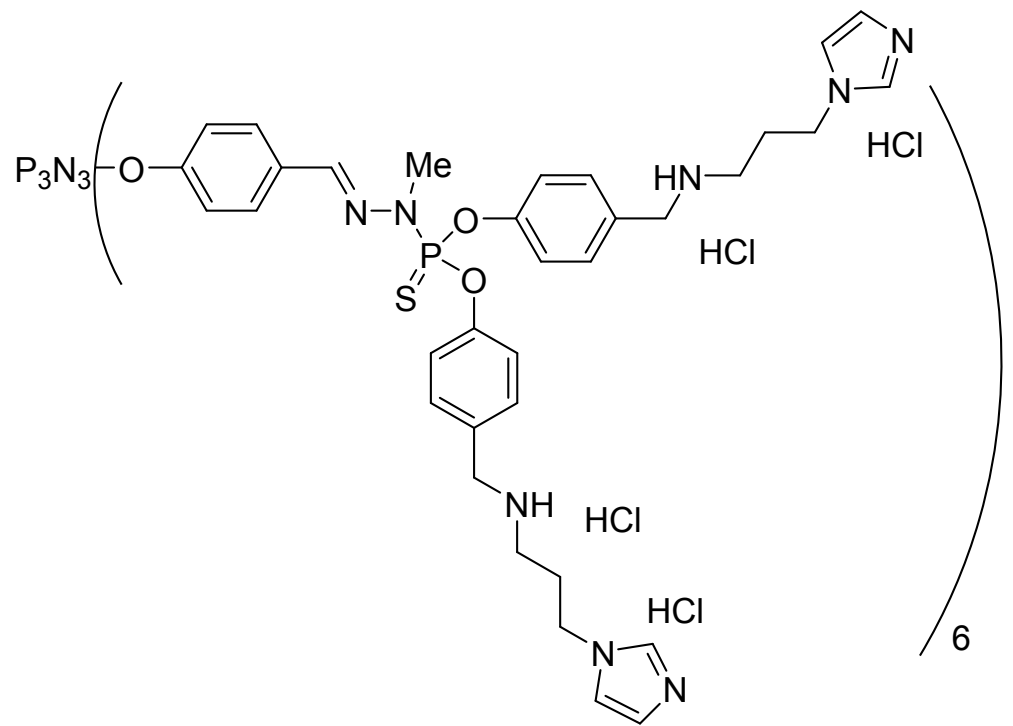

Molecular formula: $\mathrm{C}_{204} \mathrm{H}_{264} \mathrm{Cl}_{24} \mathrm{~N}_{39} \mathrm{O}_{18} \mathrm{P}_{9} \mathrm{~S}_{6}$, MW: 4873 .

\section{Compound 13G1}

$\{[\{O, O-4-(((2-($ imidazoly-1-yl)propyl)amino)methyl)phenoxy $\}$-phosphonothioate $]-\{4-((2-m e t h y l h y d r a z o n o) m e t h y l)\}$ phenoxy $\}-$ 1,3,5,2,4,6-triazatriphosphorine 


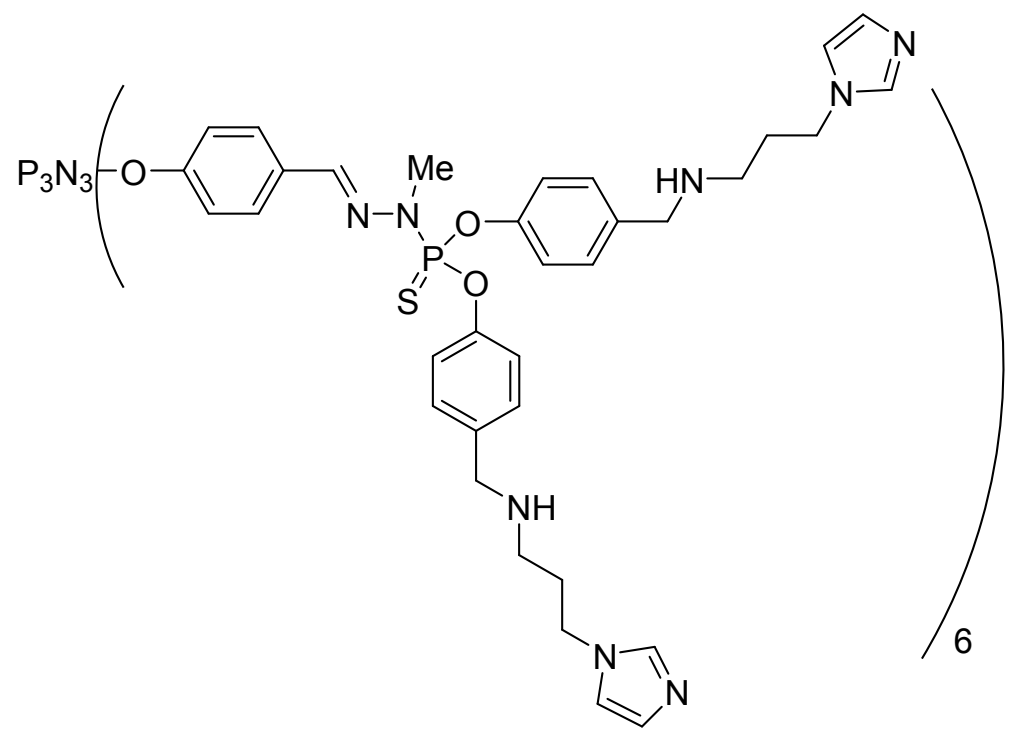

Molecular formula: $\mathrm{C}_{204} \mathrm{H}_{240} \mathrm{~N}_{51} \mathrm{O}_{18} \mathrm{P}_{9} \mathrm{~S}_{6}, \mathrm{MW}: 4166 .{ }^{1} \mathrm{H}$ NMR $(400 \mathrm{MHz}, \mathrm{CDCl} 3) \delta=7.61(\mathrm{~s}, 6 \mathrm{H}), 7.60(\mathrm{~d}, \mathrm{~J}=8 \mathrm{~Hz}, 12 \mathrm{H}), 7.41(\mathrm{~s}$, $12 \mathrm{H}), 7.21(\mathrm{~d}, \mathrm{~J}=8 \mathrm{~Hz}, 24 \mathrm{H}), 7.12(\mathrm{~d}, \mathrm{~J}=8 \mathrm{~Hz}, 24 \mathrm{H}), 7.00(\mathrm{~d}, \mathrm{~J}=4 \mathrm{~Hz}, 12 \mathrm{H}), 6.95(\mathrm{~d}, \mathrm{~J}=8 \mathrm{~Hz}, 12 \mathrm{H}), 6.85(\mathrm{brs}, 12 \mathrm{H}), 3.98(\mathrm{t}, \mathrm{J}=4$ $\mathrm{Hz}, 24 \mathrm{H}), 3.65(\mathrm{~s}, 24 \mathrm{H}), 3.23(\mathrm{~d}, \mathrm{~J}=12 \mathrm{~Hz}, 18 \mathrm{H}), 2.55(\mathrm{t}, \mathrm{J}=8 \mathrm{~Hz}, 24 \mathrm{H}), 1.90-1.84(\mathrm{~m}, 24 \mathrm{H}) .($ Yield $=76.4 \%) ;{ }^{31} \mathrm{P}\left\{{ }^{1} \mathrm{H}\right\} \mathrm{NMR}(161$ $\mathrm{MHz}, \mathrm{CDCl} 3) \delta=62.83,8.52 ;{ }^{13} \mathrm{C}\left\{{ }^{1} \mathrm{H}\right\} \mathrm{NMR}(100 \mathrm{MHz}, \mathrm{CDCl} 3) \delta=151.13,149.52(\mathrm{~d}, \mathrm{~J}=8 \mathrm{~Hz}) 138.60(\mathrm{~d}, \mathrm{~J}=17 \mathrm{~Hz}), 137.56$, 137.17, 132.26, 129.37, 129.16, 128.27, 121.35, 121.23 (d, J = $5 \mathrm{~Hz}) 118.86,53.21,45.79,44.59,33.05(\mathrm{~d}, \mathrm{~J}=11 \mathrm{~Hz}) 31.28$

\section{Compound 16G1, ${ }_{\text {HCl: }}$}

$\{[\{O, O-4-(((3-(2-m e t h y l p i p e r i d i n-1-y l) p r o p y l) a m i n o) m e t h y l) p h e n o x y\}-p h o s p h o n o t h i o a t e]-\{4-((2-$ methylhydrazono)methyl) $\}$ phenoxy $\}-1,3,5,2,4,6$-triazatriphosphorine, tetracosa (24) hydrochloride 


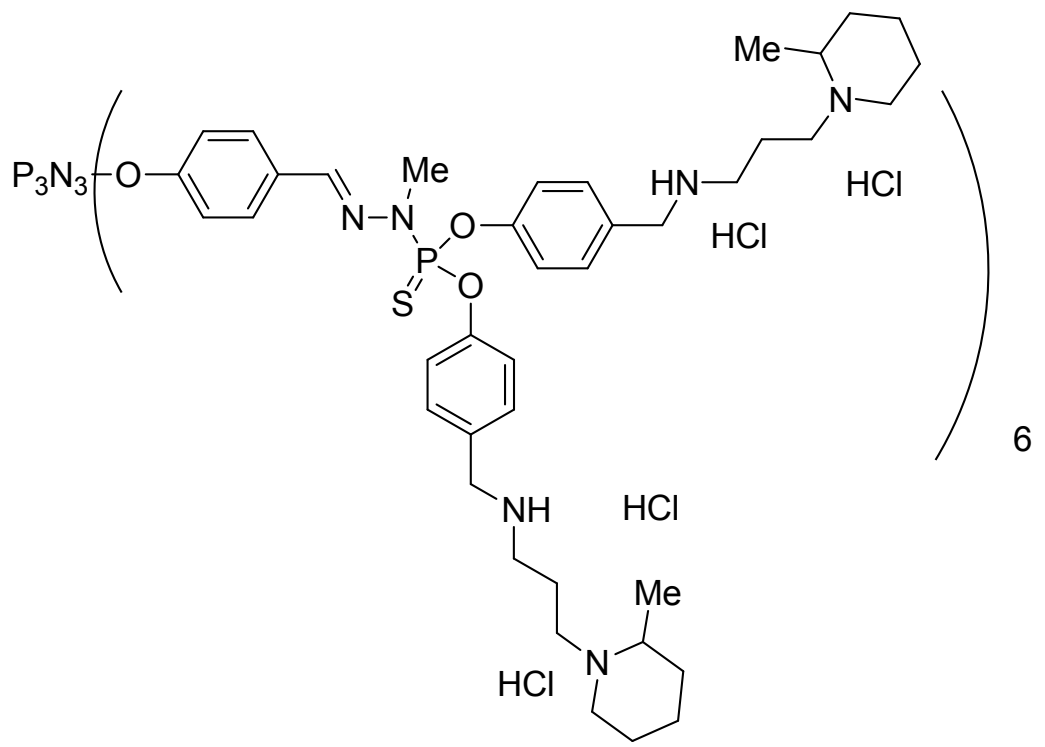

Molecular formula: $\mathrm{C}_{240} \mathrm{H}_{372} \mathrm{Cl}_{24} \mathrm{~N}_{39} \mathrm{O}_{18} \mathrm{P}_{9} \mathrm{~S}_{6}, \mathrm{MW}: 5414$.

\section{Compound 16G1}

$\{[\{O, O-4-(((3-(2-m e t h y l p i p e r i d i n-1-y l) p r o p y l) a m i n o) m e t h y l) p h e n o x y\}-p h o s p h o n o t h i o a t e]-\{4-((2-$ methylhydrazono)methyl)\}phenoxy $\}$-1,3,5,2,4,6-triazatriphosphorine 


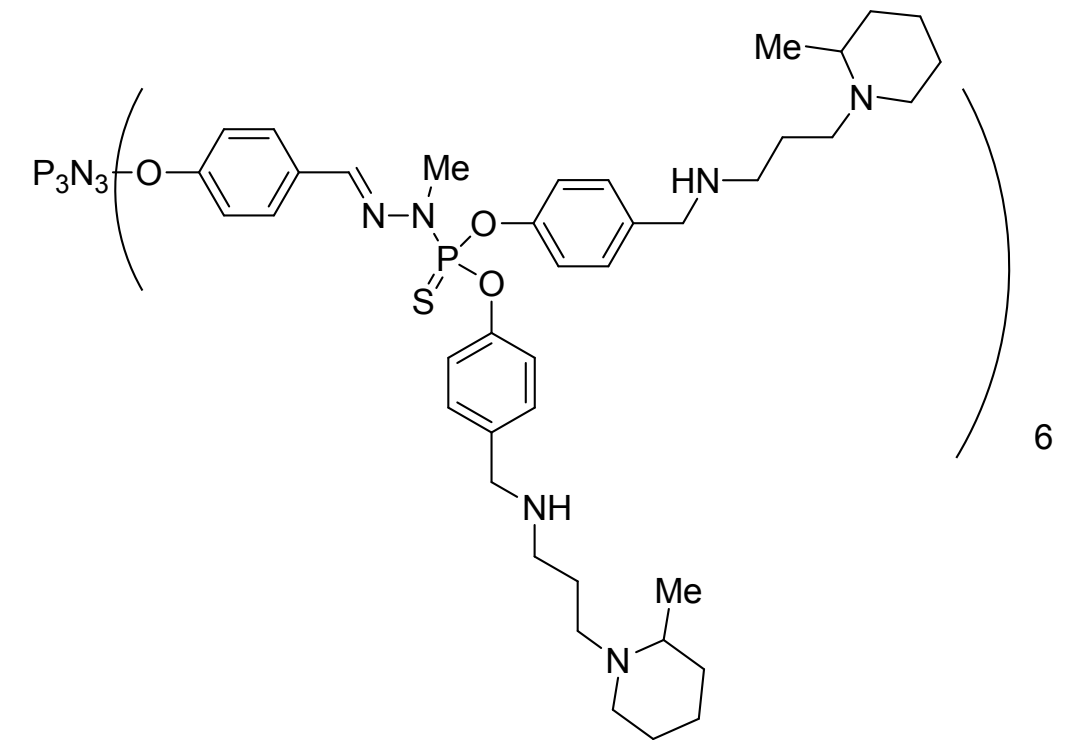

Molecular formula: $\mathrm{C}_{240} \mathrm{H}_{348} \mathrm{~N}_{39} \mathrm{O}_{18} \mathrm{P}_{9} \mathrm{~S}_{6}, \mathrm{MW}: 4539 .{ }^{1} \mathrm{H}$ NMR $(300 \mathrm{MHz}, \mathrm{CDCl} 3) \delta=7.64(\mathrm{~s}, 6 \mathrm{H}), 7.63(\mathrm{~d}, \mathrm{~J}=9 \mathrm{~Hz}, 12 \mathrm{H}), 7.31(\mathrm{~d}, \mathrm{~J}=$ $8 \mathrm{~Hz},(24 \mathrm{H}), 7.13(\mathrm{~d}, \mathrm{~J}=8 \mathrm{~Hz}, 24 \mathrm{H}), 7.00(\mathrm{~d}, \mathrm{~J}=9 \mathrm{~Hz}, 12 \mathrm{H}), 4.75(\mathrm{brs}, 12 \mathrm{H}), 3.76(\mathrm{~m}, 24 \mathrm{H}), 3.25(\mathrm{~d}, \mathrm{~J}=12 \mathrm{~Hz}, 18 \mathrm{H}), 3.00-2.90(\mathrm{~m}$, $12 \mathrm{H}), 2.90-2.80(\mathrm{~m}, 12 \mathrm{H}), 2.80-2.65(\mathrm{~m}, 24 \mathrm{H}), 2.53-2.44(\mathrm{~m}, 24 \mathrm{H}), 2.28-2.22(\mathrm{~m}, 12 \mathrm{H}), 1.88-1.83(\mathrm{~m}, 24 \mathrm{H}), 1.77-1.53(\mathrm{~m}, 48 \mathrm{H}), 1.36-$ $1.30(\mathrm{~m}, 24 \mathrm{H}), 1.10(\mathrm{~d}, \mathrm{~J}=6 \mathrm{~Hz}, 36 \mathrm{H})$. (Yield = 88\%); ${ }^{11} \mathrm{P}\left\{{ }^{1} \mathrm{H}\right\} \mathrm{NMR}(121 \mathrm{MHz}, \mathrm{CDCl} 3) \delta=62.73,8.37(\mathrm{~s}) ;{ }^{13} \mathrm{C}\left\{{ }^{1} \mathrm{H}\right\} \mathrm{NMR}(75 \mathrm{MHz}$, $\mathrm{CDCl} 3) \delta=151.23(\mathrm{~d}, \mathrm{~J}=7 \mathrm{~Hz}), 149.80$ (d, J = $9 \mathrm{~Hz}), 138.80$ (d, J = $16 \mathrm{~Hz}), 135.87,132.17,129.67,128.31,121.49,121.36$ (d, J = $5 \mathrm{~Hz}), 56.42,52.63,52.15,51.48,48.04,33.52,33.06$ (d, J = $16 \mathrm{~Hz}), 25.15,24.34,23.01,18.20$.

\section{Compound 17G1, $\mathrm{HCl}$}

$\{[\{O, O-4-(((3-(2-m e t h y l-i m i d a z o l-1-y l) p r o p y l) a m i n o) m e t h y l) p h e n o x y\}-p h o s p h o n o t h i o a t e]-\{4-((2-$ methylhydrazono)methyl) $\}$ phenoxy $\}$-1,3,5,2,4,6-triazatriphosphorine, tetracosa (24) hydrochloride 


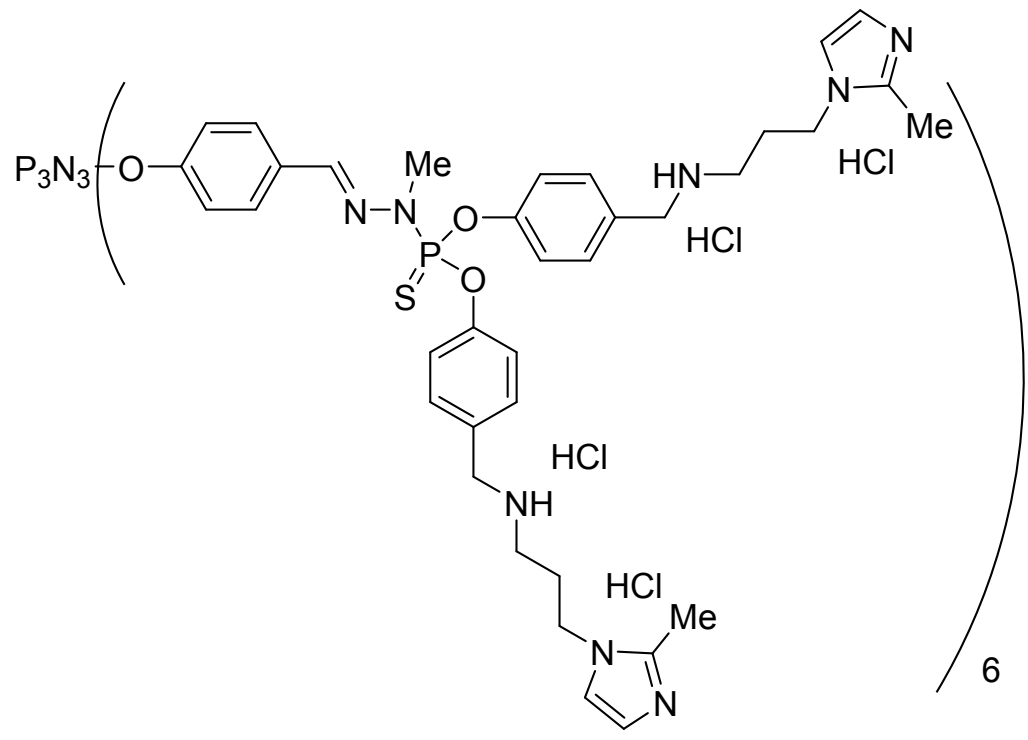

Molecular formula: ${ }_{12} \mathrm{C}_{216} \mathrm{H}_{288} \mathrm{Cl}_{24} \mathrm{~N}_{51} \mathrm{O}_{18} \mathrm{P}_{9} \mathrm{~S}_{6}$, MW: 5209 .

\section{Compound 17G1}

$\{[\{O, O-4-(((3-(2-m e t h y l-i m i d a z o l-1-y l)$ propyl)amino)methyl)phenoxy $\}$-phosphonothioate $]-\{4-((2-$ methylhydrazono)methyl)\}phenoxy\}-1,3,5,2,4,6-triazatriphosphorine 


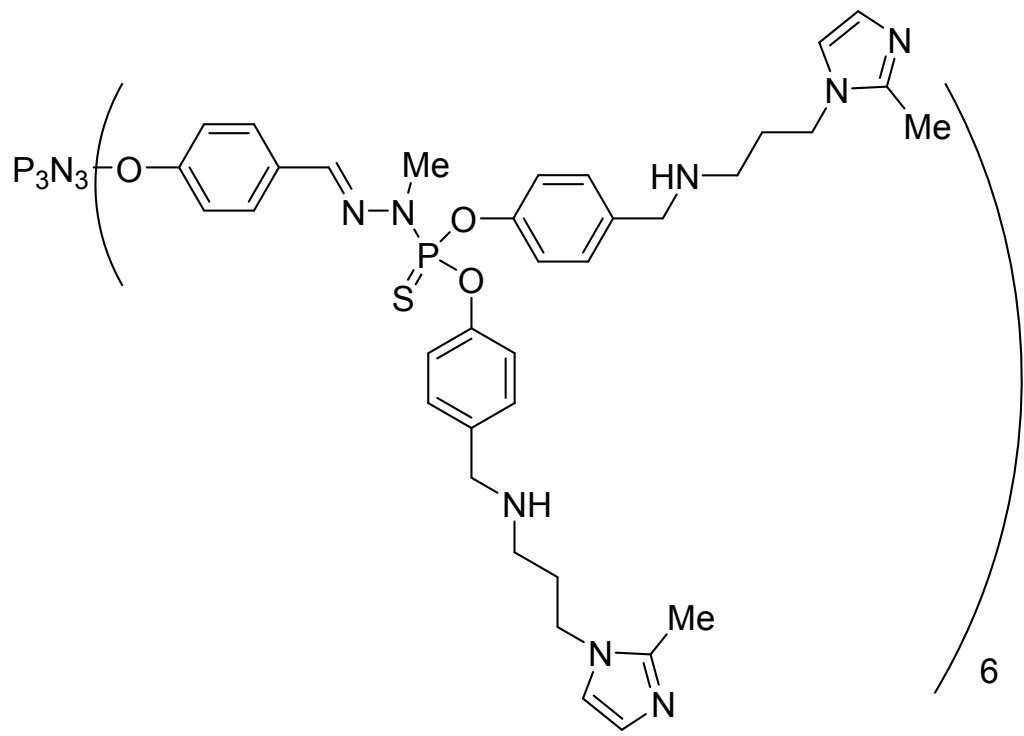

Molecular formula: $\mathrm{C}_{216} \mathrm{H}_{264} \mathrm{~N}_{51} \mathrm{O}_{18} \mathrm{P}_{9} \mathrm{~S}_{6}, \mathrm{MW}: 4334 .{ }^{1} \mathrm{H}$ NMR $(300 \mathrm{MHz}, \mathrm{CDCl}) \delta=7.61(\mathrm{~s}, 6 \mathrm{H}), 7.60(\mathrm{~d}, \mathrm{~J}=8 \mathrm{~Hz}, 12 \mathrm{H}), 7.21(\mathrm{~d}, \mathrm{~J}=$ $8 \mathrm{~Hz}, 24 \mathrm{H}), 7.13(\mathrm{~d}, \mathrm{~J}=8 \mathrm{~Hz}, 24 \mathrm{H}), 6.96(\mathrm{~d}, \mathrm{~J}=8 \mathrm{~Hz}, 12 \mathrm{H}), 6.86-6.85(\mathrm{~m}, 12 \mathrm{H}), 6.76-6.64(\mathrm{~m}, 12 \mathrm{H}), 3.89(\mathrm{t}, \mathrm{J}=9 \mathrm{~Hz}, 24 \mathrm{H}), 3.66(\mathrm{~s}$, 24H), $3.24(\mathrm{~d}, \mathrm{~J}=12 \mathrm{~Hz}, 18 \mathrm{H}), 2.56(\mathrm{t}, \mathrm{J}=9 \mathrm{~Hz}, 24 \mathrm{H}), 2.33(\mathrm{~s}, 36 \mathrm{H}), 1.89-1.75(24 \mathrm{H}) .(Y i e l d=76 \%) ;{ }^{31} \mathrm{P}\left\{{ }^{1} \mathrm{H}\right\} \mathrm{NMR}(121 \mathrm{MHz}$, $\mathrm{CDCl} 3) \delta=62.80,8.50 ;{ }^{13} \mathrm{C}\left\{{ }^{1} \mathrm{H}\right\} \operatorname{NMR}(75 \mathrm{MHz}, \mathrm{CD} 2 \mathrm{Cl} 3) \delta=151.09,149.52(\mathrm{~d}, \mathrm{~J}=7 \mathrm{~Hz}), 144.43,138.52(\mathrm{~d}, \mathrm{~J}=17 \mathrm{~Hz}), 137.57$, 132.27, 129.18, 128.27, 127.11, 121.30, 121.23 (d, J = $5 \mathrm{~Hz}), 119.09,53.26,45.84,43.62,33.04$ (d, J = $12 \mathrm{~Hz}), 30.95,13.01$

Molecular modeling studies 


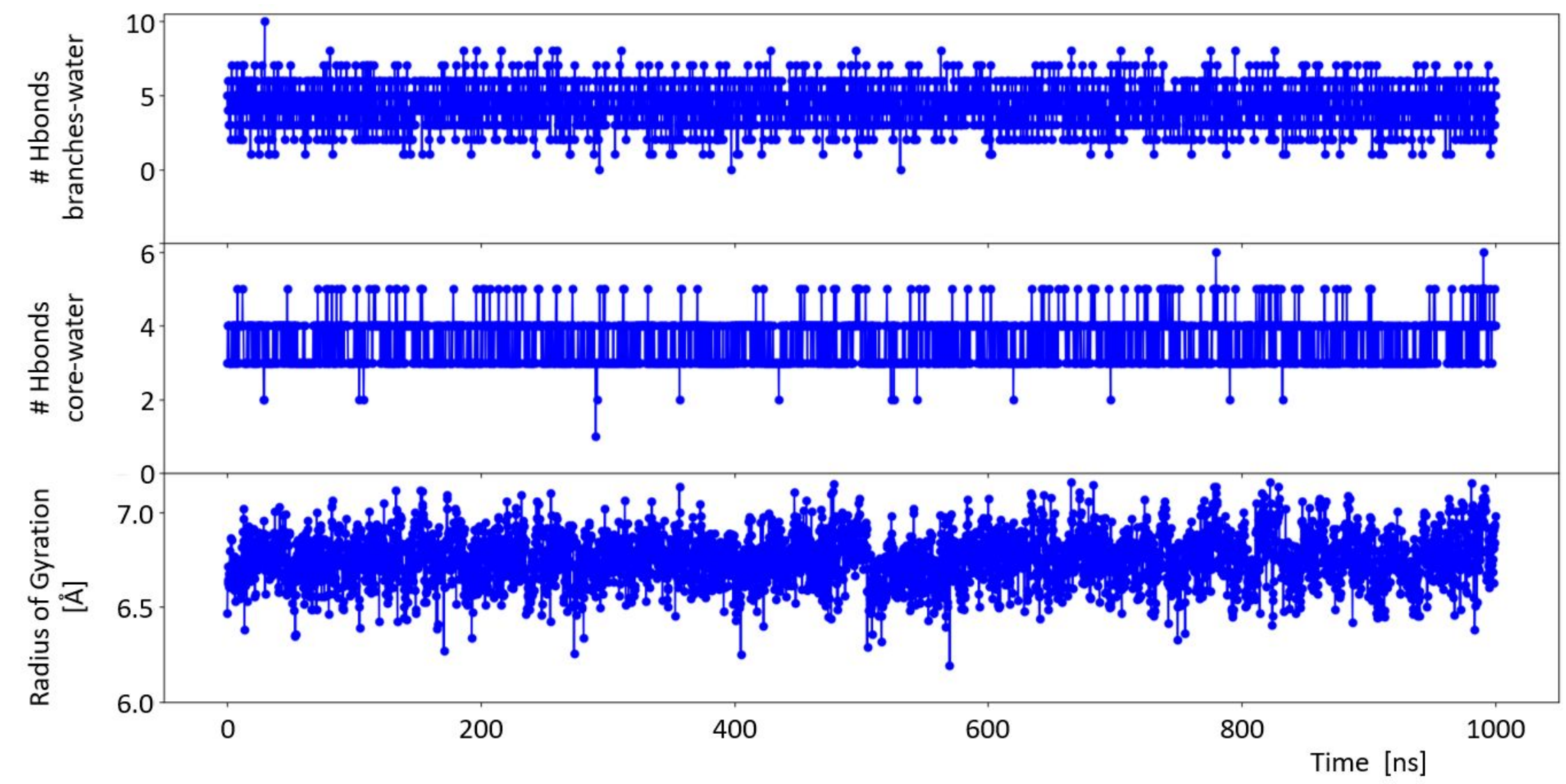


Figure F5: $1 \mathbf{G O}_{\text {, } \mathrm{HCl}}$ : statistics of hydrogen bonds of branches and core with water molecules and of the radius of gyration versus simulation time on the top, in the middle, and at the bottom, respectively.

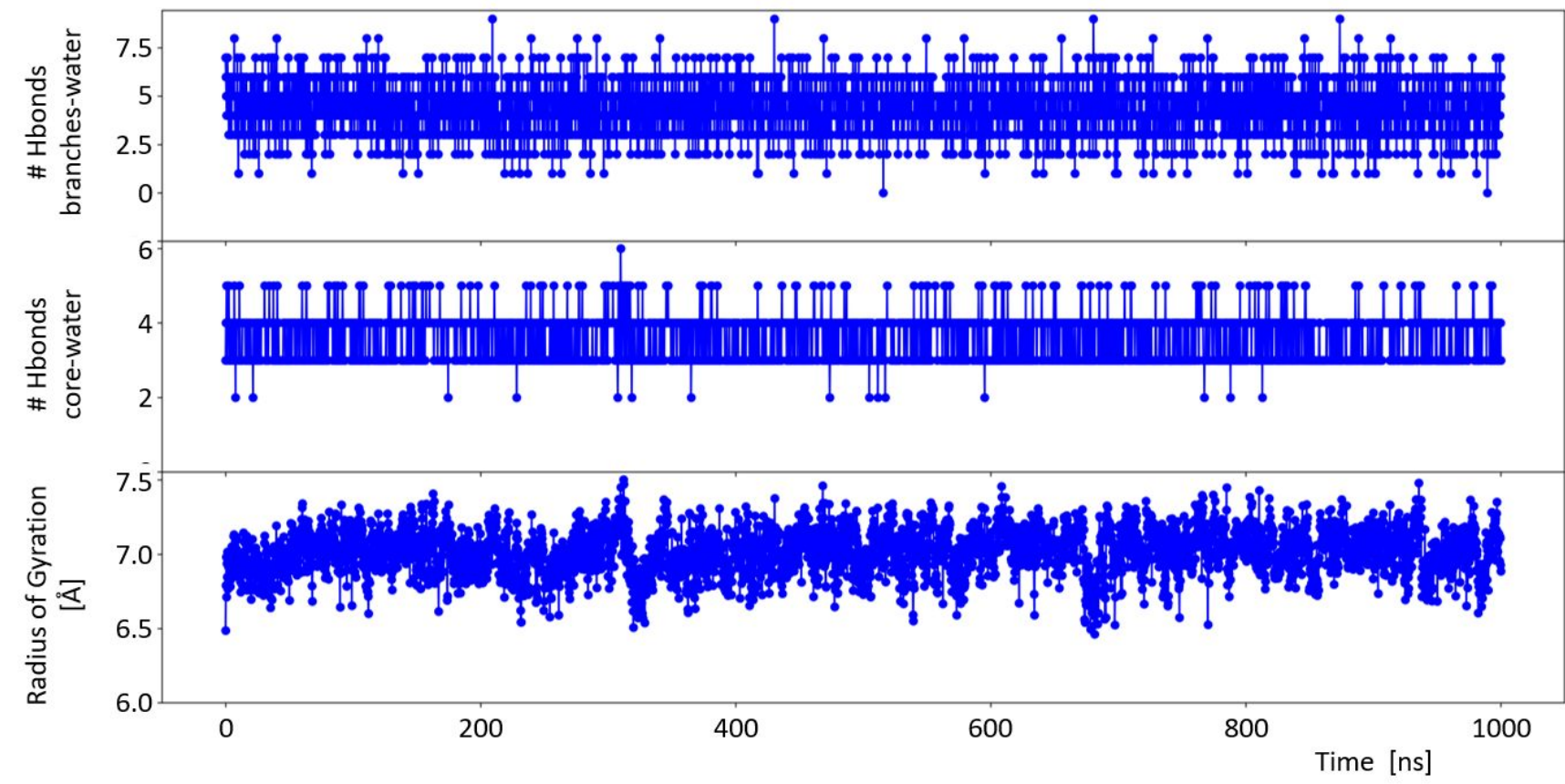

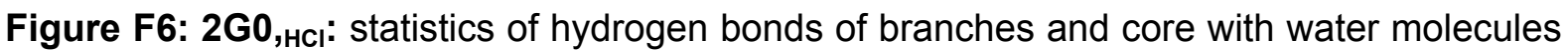
and of the radius of gyration versus simulation time on the top, in the middle, and at the bottom, respectively.

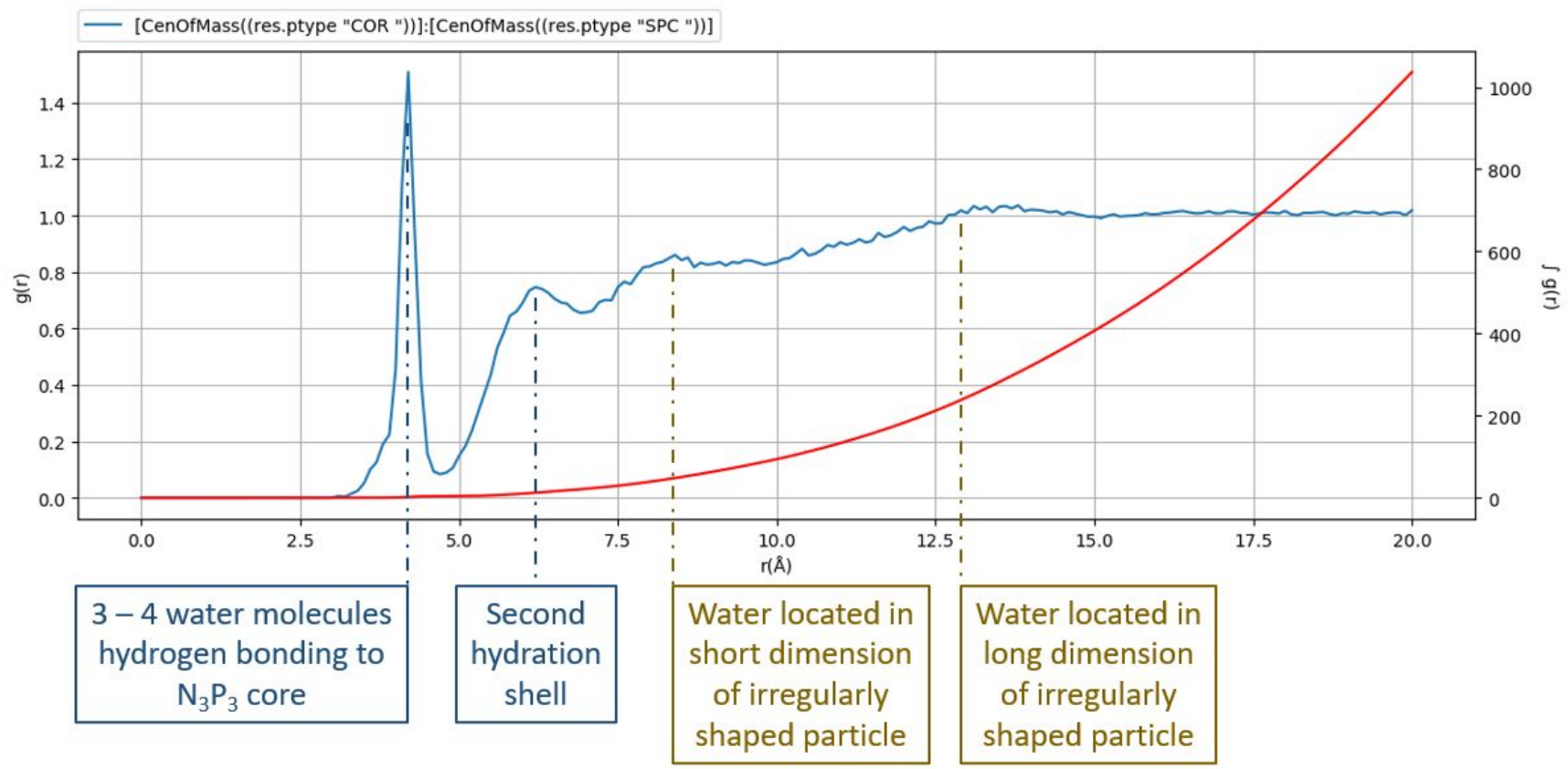

Figure F7: 1G0, ${ }_{\mathrm{HCl}}$ : Radial distribution function of water around N3P3 core. $1 \mathrm{G} 0, \mathrm{HCl}$ appears as an irregularly shaped nanoparticle of changing form throughout the molecular dynamics simulation. The core hydrogen bonds 1 to 6 water molecules, typically 3 to 4 (Supplements Figure F5). The shorter dimension of the dendrimer is about $15 \AA$, the longer one about $24 \AA$. The peaks of first and second hydration shells of the core, as well as the 
shorter and longer dimensions fit well to a radius of gyration of 6.5 to $7 \AA$ (Supplements Figure F5).

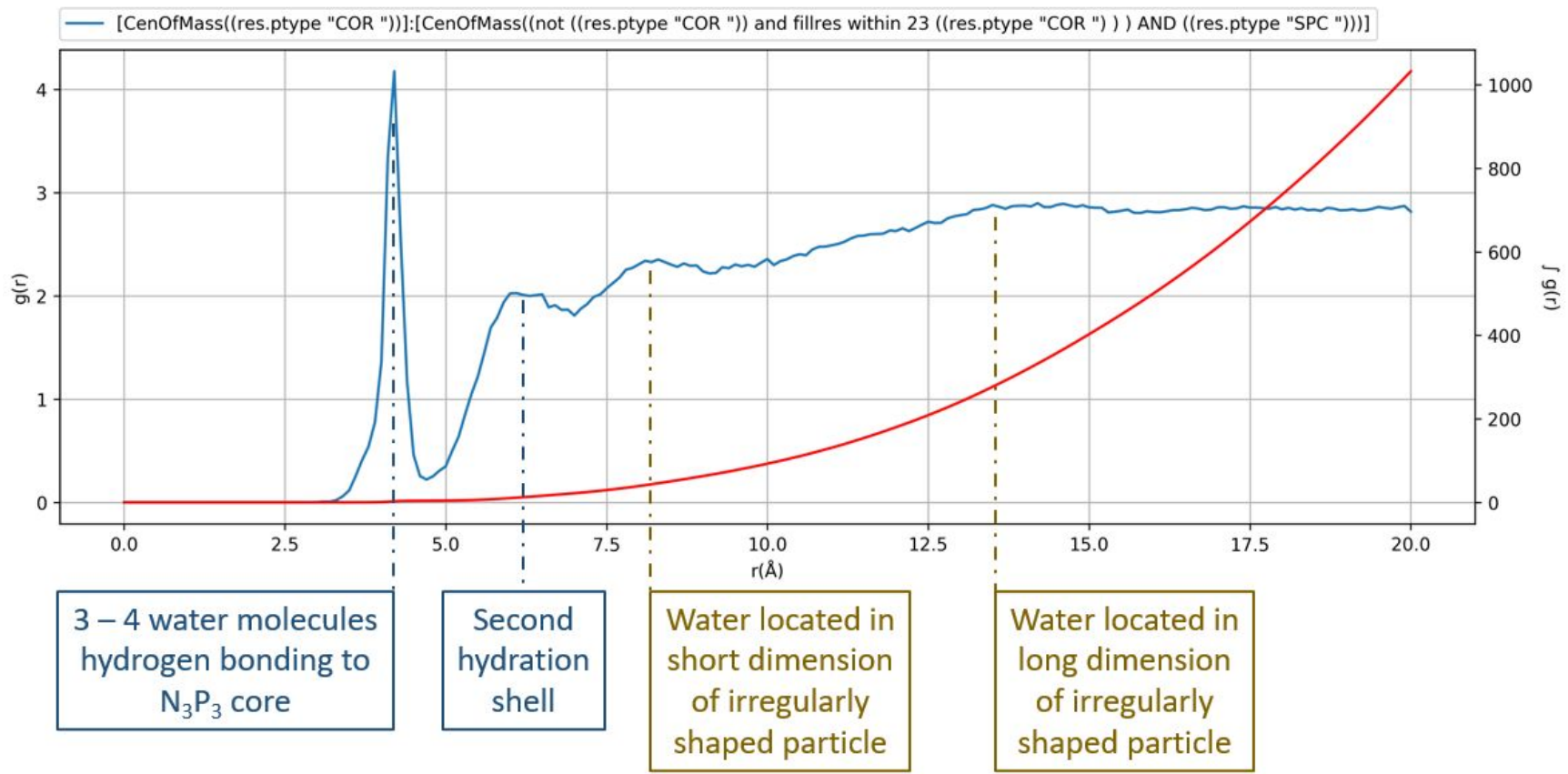

Figure F8: 2G0, ${ }_{\mathrm{HCl}}$ : Radial distribution function of water around N3P3 core. $2 \mathrm{G} 0, \mathrm{HCl}$ appears as an irregularly shaped nanoparticle of changing form throughout the molecular dynamics simulation. The core hydrogen bonds 2 to 6 water molecules, typically 3 to 4 (Supplements Figure F6). The shorter dimension of the dendrimer is about $14 \AA$, the longer one about $25 \AA$. The peaks of first and second hydration shells of the core, as well as the shorter and longer dimensions fit well to a radius of gyration of 6.7 to $7.4 \AA$ (Supplements Figure F6). 

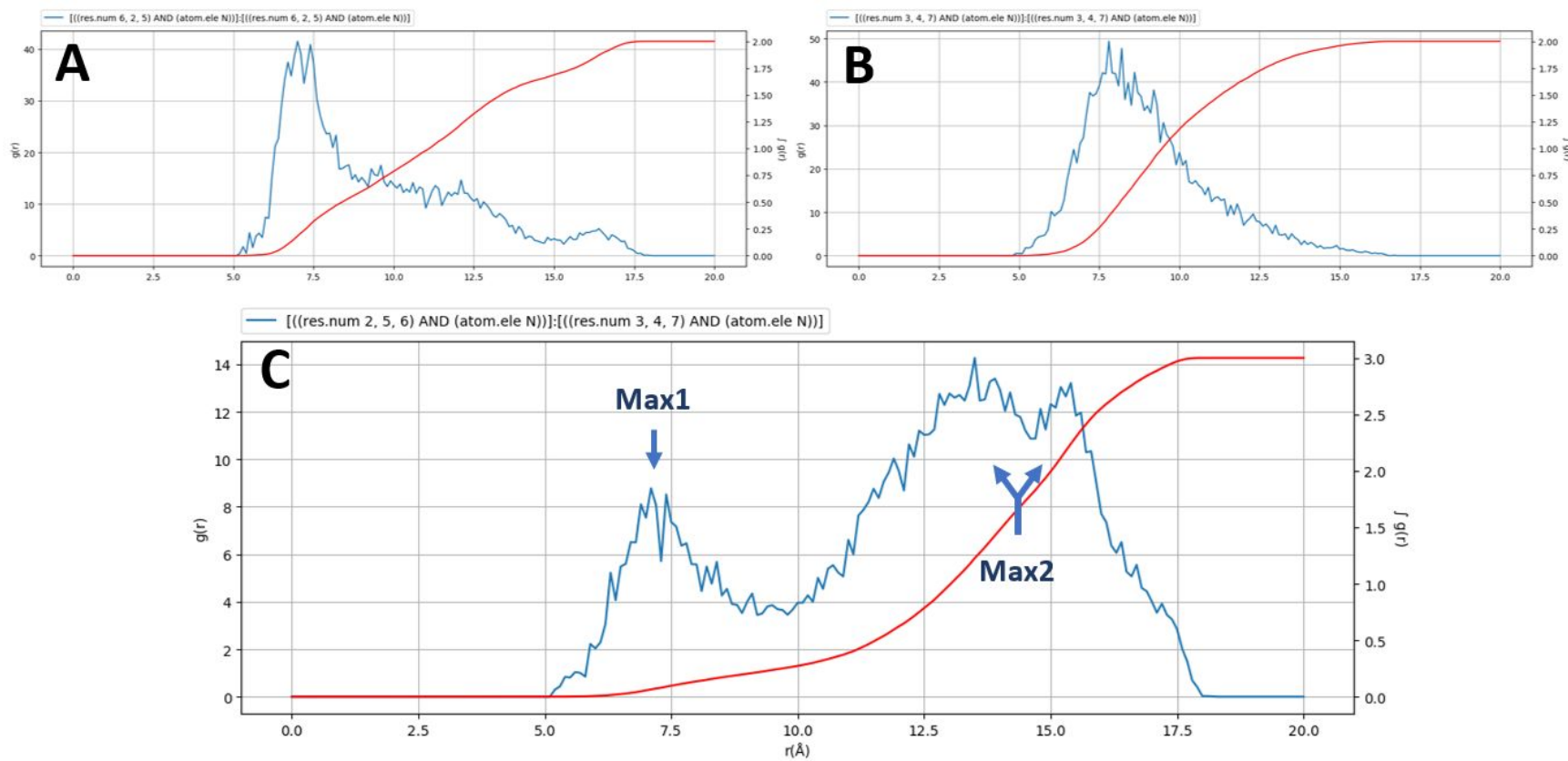

Figure F9: 1G0, , $_{\text {: }}$ : Radial distribution function of the protonated tertiary nitrogen atoms of the dendrimer branches. Analysis of branches above or below the plane of the N3P3 core in A) and B), respectively, of branches above versus below in $C$ ). The nitrogen atoms tend to be at a distance of $7.5 \AA$ in $A$ ) and $B$ ) as indicated by the maxima of the $g(r)$ curve in blue, which corresponds to branch conformations that extend roughly perpendicularly to the N3P3 plane. These conformations give rise to the split maximum Max2 of the $g(r)$ curve around 14 $\AA$ in $\mathrm{C}$ ). The maximum Max 1 of $\mathrm{g}(\mathrm{r})$ around $7 \AA$ in $\mathrm{C}$ ) arises from branch conformations that strongly tilt towards the N3P3 plane and approach nitrogen atoms of branches above and below the plane. Branch conformations are highly mobile switching frequently from perpendicular to tilted and back, thereby distributing the positively charged nitrogen atoms evenly around the core and optimizing the electrostatic repulsion between the charged groups. Transient interactions of chloride ions and positively charged nitrogen atoms are also observed. 

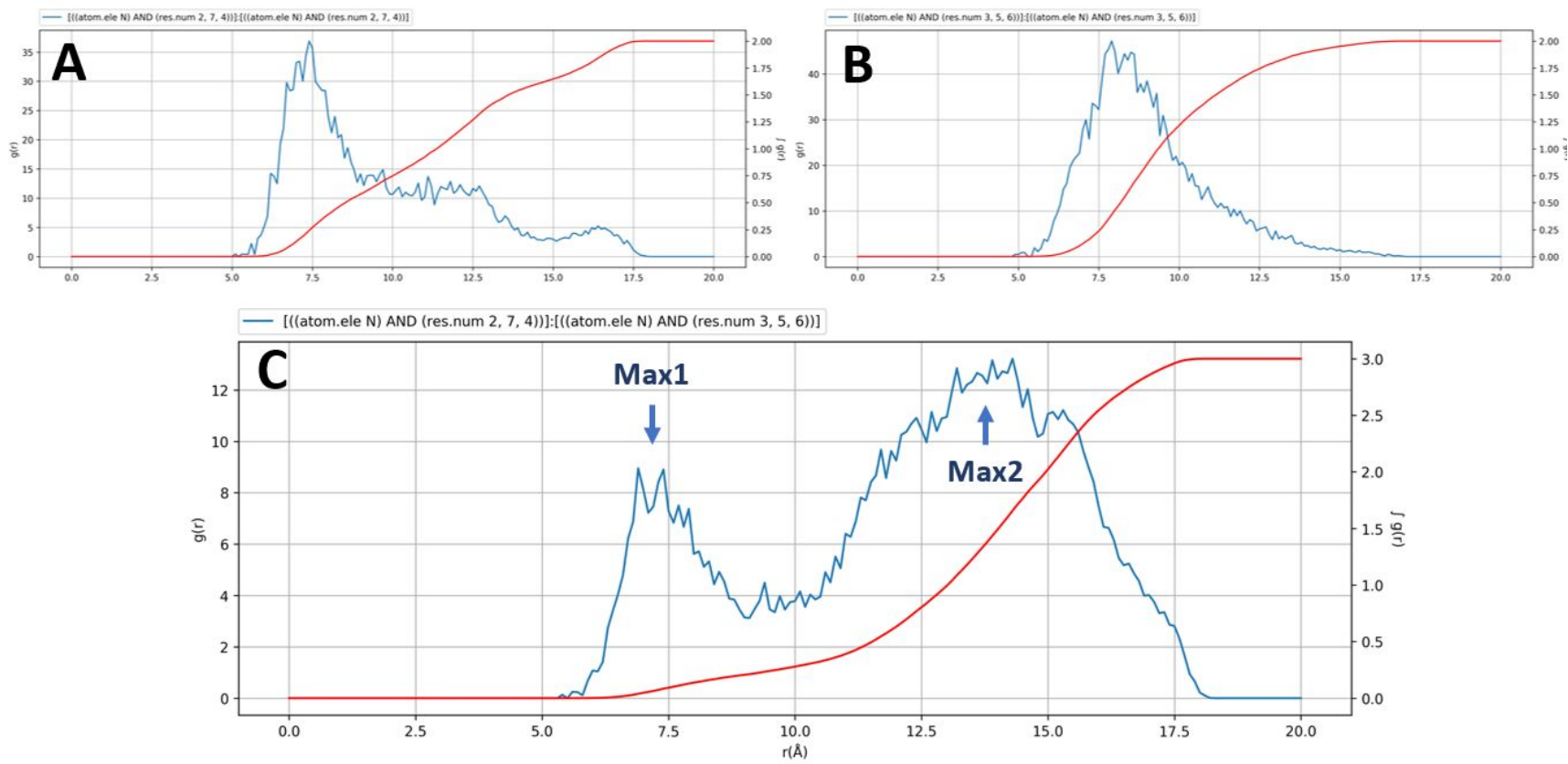

Figure F10: 2G0, , $_{\text {: }}$ : Radial distribution function of the protonated tertiary nitrogen atoms of the dendrimer branches. Analysis of branches above or below the plane of the N3P3 core in $A$ ) and $B$ ), respectively, of branches above versus below in $C$ ). The nitrogen atoms tend to be at a distance of $7.5 \AA$ in $A$ ) and $B$ ) as indicated by the maxima of the $g(r)$ curve in blue, which corresponds to branch conformations that extend roughly perpendicularly to the N3P3 plane. These conformations give rise to the maximum Max2 of the $g(r)$ curve around $14 \AA$ in C). The maximum Max1 of $g(r)$ around $7.5 \AA$ in $C$ ) arises from branch conformations that tilt strongly towards the N3P3 plane and approach nitrogen atoms of branches above and below the plane. Again, branch conformations are highly mobile switching frequently from perpendicular to tilted and back, thereby distributing the positively charged nitrogen atoms evenly around the core and optimizing the electrostatic repulsion between the charged groups. Transient interactions of chloride ions and positively charged nitrogen atoms are also observed. 